\title{
GLOBAL FAMILIES OF LIMIT CYCLES OF PLANAR ANALYTIC SYSTEMS
}

\author{
L. M. PERKO
}

\begin{abstract}
The global behavior of any one-parameter family of limit cycles of a planar analytic system $\dot{x}=f(x, \lambda)$ depending on a parameter $\lambda \in R$ is determined. It is shown that any one-parameter family of limit cycles belongs to a maximal one-parameter family which is either open or cyclic. If the family is open, then it terminates as the parameter or the orbits become unbounded, or it terminates at a critical point or on a (compound) separatrix cycle of the system. This implies that the periods in a one-parameter family of limit cycles can become unbounded only if the orbits become unbounded or if they approach a degenerate critical point or (compound) separatrix cycle of the system. This is a more specific result for planar analytic systems than Wintner's principle of natural termination for $n$-dimensional systems where the periods can become unbounded in strange ways. This work generalizes Duff's results for one-parameter families of limit cycles generated by a one-parameter family of rotated vector fields. In particular, it is shown that the behavior at a nonsingular, multiple limit cycle of any one-parameter family of limit cycles is exactly the same as the behavior at a multiple limit cycle of a one-parameter family of limit cycles generated by a one-parameter family of rotated vector fields.
\end{abstract}

\section{INTRODUCTION}

In a hallmark paper [1], Duff described the global behavior of any oneparameter family of limit cycles generated by a family of rotated vector fields. He showed that a one-parameter family of limit cycles generated by a family of rotated vector fields expands or contracts monotonically with the parameter until it intersects a critical point on the Poincare sphere; i.e., the family terminates at a single critical point of the vector field or on a (compound) separatrix cycle of the vector field on the Poincare sphere. It also follows from the results in [1] that the only possible type of bifurcation that can occur in a one-parameter family of limit cycles generated by a family of rotated vector fields is a saddle-node bifurcation at a semistable limit cycle of the family. Duff's results describing the global behavior of one-parameter families of limit cycles generated by a family of rotated vector fields have proved to be immensely useful in the study of limit cycles of planar dynamical systems. Unfortunately, up to this point, no results analogous to Duff's results for families of rotated vector fields have been

Received by the editors December 21, 1988.

1980 Mathematics Subject Classification (1985 Revision). Primary 34C05, 34C25; Secondary $34 \mathrm{C} 35$. 
available for general families of planar vector fields. In this paper we generalize Duff's results for limit cycles of families of rotated vector fields to general families of planar analytic vector fields. We show that any global family of limit cycles of a planar analytic family of vector fields is the union of a finite number of one-parameter families of limit cycles which are either cyclic or satisfy the same type of termination principle as Duff's families. In the general case the limit cycles need not expand or contract monotonically with the parameter. But we show that even this result holds in the neighborhood of any nonsingular multiple limit cycle in the family.

The purpose of this paper therefore is to describe the global behavior of the one-parameter families of limit cycles generated by a family of planar analytic systems

$$
\dot{x}=f(x, \lambda)
$$

depending on a parameter $\lambda \in \mathbf{R}$. In order to accomplish this goal, two basic questions must be answered:

1. Can a one-parameter family of limit cycles be continued through a complex bifurcation in a unique way?

2. How does a maximal one-parameter family of limit cycles, which is not cyclic, terminate?

The answer to the first question is yes, provided that the system $\left(1_{\lambda_{\lambda}}\right)$ is analytic. An example is given in this introduction which shows that the answer to the first question is no, even for $C^{\infty}$-systems. In $\S 1$ of this paper we use Puiseux series to show that, in the analytic case, the limit cycle function defined by the Poincare map can be continued in a unique way through any bifurcation point; see Lemma 1.4. Then in Lemma 1.6 we show that each branch of the limit cycle function defines an analytic path of limit cycles. This in turn determines how to uniquely continue any one-parameter family of limit cycles through a bifurcation orbit; see Definition 1.9. Wintner [2], in his 1931 study of one-parameter families of periodic orbits of the restricted three-body problem, used Puiseux series to show that, in the analytic case, any one-parameter family of periodic orbits can be uniquely continued through any bifurcation except a period-doubling bifurcation. However, Wintner bases his argument on the continuation of analytic surfaces rather than analytic paths and the details involving the use of Puiseux series are not given in [2]. Consequently, Wintner's argument, though sound, is somewhat difficult to follow. Hopefully, the detailed use of Puiseux series in describing how to uniquely continue an analytic path of periodic orbits through a bifurcation point, given in the first section of this paper, will make Wintner's idea clear. All of the arguments and results in the first part of $\S 1$, up to and including Lemma 1.5, hold for higher-dimensional systems $\left(1_{\lambda}\right)$ with $x \in \mathbf{R}^{n}$. However, in Lemma 1.6 we use the fact that trajectories of planar systems do not cross, and Lemma 1.6 and the remainder of the paper apply only to planar systems. 
The answer to the second question is the topic of the global analysis in $\S 2$ of this paper. We first show that any one-parameter family of limit cycles can be extended to a maximal one-parameter family of limit cycles which is either open or cyclic; see Theorems 1 and 2. An example of a cyclic family is given at the end of this introduction, and a one-parameter family of limit cycles, $S$, as defined in Definition 1.9, is said to be open if the domain of any path that generates $S$ is open; see Definition 1.6. The main result of this paper, describing the termination of any maximal one-parameter family of limit cycles which is not cyclic, can be stated as follows; see Theorem 3 in $\S 2$.

Planar termination principle. Any maximal one-parameter family of limit cycles of a planar analytic system $\left(1_{\lambda}\right)$ is either open or cyclic. If it is open, then it terminates as either the parameter or the orbits become unbounded; or the family terminates either at a critical point or on a (compound) separatrix cycle of $\left(1_{\lambda}\right)$.

The size or dimension of a periodic orbit is measured by its norm, defined in Definition 2.1 at the beginning of $\S 2$, and a compound separatrix cycle or graphic of $\left(1_{\lambda}\right)$ is defined as a connected union of compatibly oriented separatrix cycles; a more precise definition can be found in [3, p. 518] or in [4, p. 141]. If we consider the one-parameter family of limit cycles generated by the vector field $\left(1_{\lambda}\right)$ on the Poincare sphere, then the orbit dimensions remain bounded and the family terminates either at a critical point or (compound) separatrix cycle of $\left(1_{\lambda}\right)$ on the Poincare sphere or as the parameter becomes unbounded. The proof of the planar termination principle is based on a series of lemmas which establish that the limit set of any one-parameter family of limit cycles is either a critical point or a compound separatrix cycle of $\left(1_{\lambda}\right)$. The proofs of these lemmas have the flavor of the Poincare-Bendixson theory used to determine that the limit set of any trajectory of a planar system is either a critical point, a cycle, or a compound separatrix cycle of the system. However, there is definitely a difference between determining the limit set of a trajectory and determining the limit set of a one-parameter family of limit cycles, and the analysis in $\S 2$ necessarily requires some new ideas different from the standard Poincaré-Bendixson theory. These ideas originated in the author's paper [3] where he showed that the limit cycles of any planar analytic system can accumulate only at a critical point or on a graphic of the system on the Poincare sphere. In $\S 2$ of this paper we extend these ideas to show that any one-parameter family of limit cycles terminates at a critical point or graphic of the system on the Poincare sphere. It also follows immediately from Theorem 3 in [3] that if a one-parameter family of periodic orbits or cycles of $\left(1_{\lambda}\right)$ consists of a continuous band of cycles, then the inner and outer boundaries of this continuous band of cycles each consists of either a critical point or graphic of $\left(1_{\lambda}\right)$ on the Poincare sphere.

Just as the Poincaré-Bendixson theorem gives us explicit information on the nature of the limit set of any trajectory of a planar system, the planar termination principle established in this paper gives us explicit information on how any one-parameter family of limit cycles of a planar analytic system $\left(1_{\lambda}\right)$ 
terminates. This result should be compared with the more general result for higher dimensional systems established by Wintner in [2].

Wintner's principle of natural termin.tion. Any maximal one-parameter family of periodic orbits of analytic system $\left(1_{\lambda}\right)$ with $x \in \mathbf{R}^{n}$ is cyclic or terminates as either the periods, the parameter, or the orbits become unbounded, or the family terminates at an equilibrium point or at a period-doubling bifurcation orbit of $\left(1, \lambda_{i}\right.$.

In particular, this result points out that for higher dimensional systems with $n \geq 3$, a family of periodic orbits can terminate at a period-doubling bifurcation. This cannot happen in planar systems since trajectories do not cross. Several examples of period-doubling bifurcations are contained in Sparrow's work [5] on the Lorenz system. Also, for $n \geq 3$, the periods in a one-parameter family can become unbounded in strange ways; for example, the periodic orbits may belong to a strange invariant set generated at a bifurcation value for which there is a homoclinic tangency of the stable and unstable manifolds of the Poincaré map. This cannot happen for planar systems. In fact, for planar systems we have the following result.

Theorem. The periods of a bounded, one-parameter family of limit cycles of a planar analytic system $\left(1_{\lambda}\right)$ can become unbounded only if the limit cycles in the family approach a degenerate critical point or (compound) separatrix cycle of the system.

The proof of this theorem follows immediately from the planar termination principle and the fact that if the limit cycles in the family approach a critical point $x_{0}$ of the system, then the periods remain bounded unless $x_{0}$ is a degenerate critical point of the system $\left(1_{i_{0}}\right)$, i.e., unless det $\operatorname{Df}\left(x_{0}, \lambda_{0}\right)=0$. This latter fact follows from Lemmas 1 and 2 in [4] or from the results in [6].

Besides the planar termination principle, there are several other results contained in this paper which generalize Duff's results for families of rotated vector fields in [1]. These results are developed in the appendix at the end of this paper. One of the results is of particular interest, since it describes the behavior of a one-parameter family of limit cycles in the neighborhood of a nonsingular multiple limit cycle (defined in the appendix) and since bifurcations occur only at multiple limit cycles of $\left(1_{i}\right)$ or at cycles of $\left(1_{i}\right)$ which belong to continuous bands of cycles.

Theorem (Behavior at a Nonsingular Multiple Limit Cycle). If $L_{0}$ is a nonsingular, multiple limit cycle of $\left(1_{i_{0}}\right)$, then $L_{0}$ belongs to a unique one-parameter family of limit cycles of $\left(1_{\lambda}\right)$; furthermore,

(1) if the multiplicity of $L_{0}$ is odd, then the family either expands or contracts monotonically as $\lambda$ increases through $\lambda_{0}$, and

(2) if the multiplicity of $L_{0}$ is even, then $L_{0}$ bifurcates into a stable and an unstable limit cycle as $\lambda$ varies from $\lambda_{0}$ in one sense and $L_{0}$ disappears 
as $\lambda$ varies from $\lambda_{0}$ in the opposite sense; i.e., there is a saddle-node bifurcation at $\lambda_{0}$.

The variation of $\lambda$ which causes the bifurcation in case (2) and which determines whether the family expands or contracts in case (1) is described at the end of the appendix. These results generalize the corresponding results for families of rotated vector fields contained in $[1,6,7]$.

Before giving some examples which illustrate the various types of bifurcations that can occur in systems of the form $\left(1_{\lambda}\right)$, we point out one more important termination principle which has been established by Mallet-Paret and Yorke [8] for higher dimensional generic systems where only period-multiplying bifurcations occur. For $C^{\infty}$-systems they have established that any one-parameter family of periodic orbits of a generic system can be continued in a unique way through any period-multiplying bifurcation, except for a period-doubling bifurcation, simply by requiring the period to vary continuously. As in Wintner [2], the family is said to terminate at a period-doubling bifurcation; see [8, p. 435]. Furthermore, Mallet-Paret and Yorke [8] show that any maximal, oriented, one-parameter family of periodic orbits of a generic system is either open or cyclic. If it is open, then it terminates as either the periods, the parameter, or the orbit dimensions become unbounded; or the family terminates at a Hopf bifurcation at an equilibrium point of the system. This is referred to as the snake termination principle in [8]. A snake is defined in [8] as a maximal, oriented, one-parameter family of periodic orbits. It should also be pointed out that there are several results for nongeneric $C^{1}$-systems established in [9] and [10]. In particular, Alligood and Yorke [9] show that any non-Möbius orbit which has no multipliers that are roots of unity is globally continuable; see [ 9 , p. 65]. In terms of the definition of global continuability, given in [9, p. 61], this result also establishes how the noncyclic branches of the global family terminate. These results on the termination of the family branches appear to be equivalent to Wintner's principle of natural termination, except that for $n>4$ it is apparently possible to have the virtual periods, defined in $[9$, p. 60], being unbounded without the periods being unbounded; see [11, p. 13].

A rich source of examples for planar systems comes from systems of the form

$$
\dot{x}=-y+x \Psi(r, \lambda), \dot{y}=x+y \Psi(r, \lambda) .
$$

This is the basic form of several of Poincaré's examples in [12]. The bifurcation diagram for this system is essentially given by the relation $\Psi(r, \lambda)=0$. For example, the above system with $\Psi(r, \lambda)=\lambda-(r-1)^{2}$ has a saddle-node or jughandle bifurcation at $r=1, \lambda=0$, as shown in Figure 1(a); see [13, p. 146]. This system with $\Psi(r, \lambda)=\left[\lambda-(r-1)^{2}\right](r-1)$ has a pitchfork bifurcation at $r=1, \lambda=0$, as shown in Figure 1(b); see [13, p. 147]. And the above system with $\Psi(r, \lambda)=\left[(\lambda-1)-(r-1)^{2}\right](r-1)(\lambda-1)$ has a more complex type of bifurcation at $r=1, \lambda=1$, as shown in Figure 1(c). In this last example, the bifurcation orbit is a circle of radius 1 , centered at the origin, which belongs to 


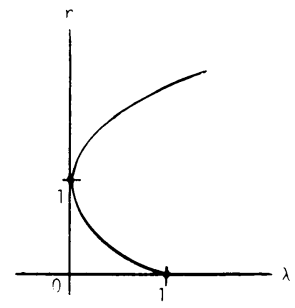

(a)

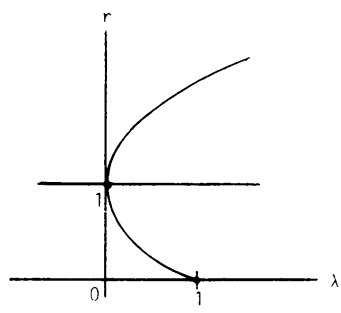

(b)

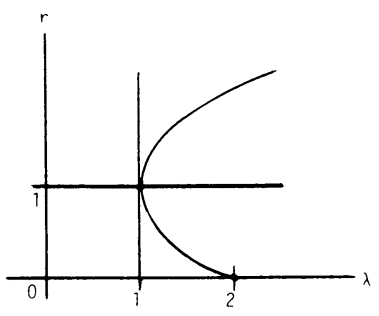

(c)

FIGURE 1. Bifurcation diagrams representing (a) a saddlenode or jug-handle bifurcation, (b) a pitchfork bifurcation, and (c) a bifurcation at a cycle belonging to a continuous band of cycles

a continuous band of cycles of the above system. This is similar to the example on p. 458 in [6].

An example of a cyclic family is furnished by the above system with $\Psi(r, \lambda)=$ $\left(r^{2}-2\right)^{2}+\lambda^{2}-1$. The bifurcation diagram for this cyclic family is shown in Figure 2. There are saddle-node bifurcations at $r=\sqrt{2}$ and $\lambda= \pm 1$. A cyclic family is any family generated by a cyclic path as defined in Definition 1.8. This definition corrects the definition of a cyclic family given in [8]. The necessity for this correction is pointed out at the beginning of $\S 2$ using the above example of a cyclic family.

An example of a $C^{\infty}$-family which cannot be continued through a bifurcation in a well-defined way is furnished by the above system with $\Psi(r, \lambda)=$ $\left[r^{2}-\phi_{+}(\lambda)\right]\left[r^{2}-\phi_{-}(\lambda)\right]$ where

$$
\phi_{ \pm}(\lambda)= \begin{cases}1 \pm \exp (-1 / \lambda) & \text { for } \lambda>0 \\ 1 & \text { for } \lambda \leq 0\end{cases}
$$

The bifurcation diagram for this system is shown in Figure 3. Clearly, the oneparameter family of limit cycles defined by this system for $\lambda \leq 0$ cannot be continued through the bifurcation at $r=1$ and $\lambda=0$ in a unique way.

Finally, an example of a family of limit cycles whose periods become unbounded as the limit cycles approach a degenerate critical point is furnished by

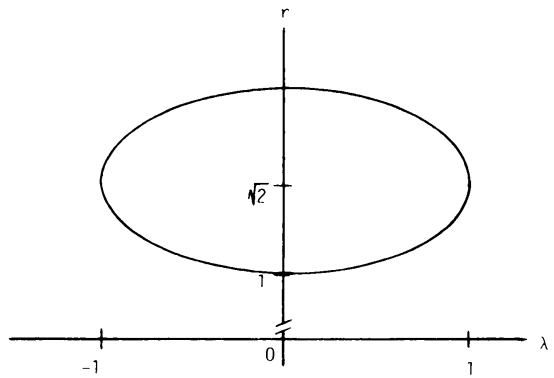

FigurE 2. The bifurcation diagram of a cyclic family. 


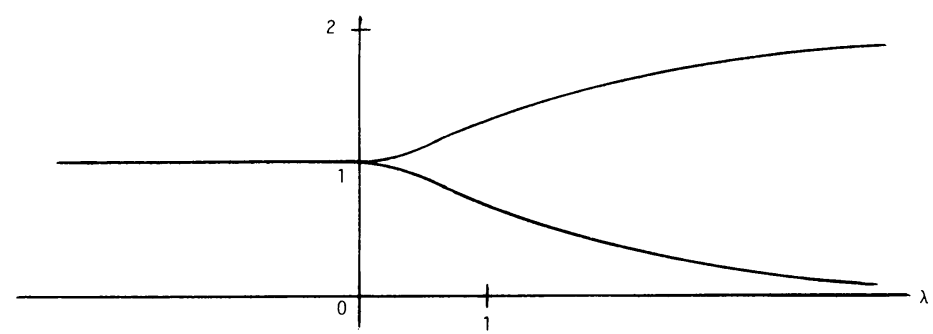

Figure 3. The bifurcation diagram of a $C^{\infty}$-family which cannot be continued through a bifurcation point in a unique way.

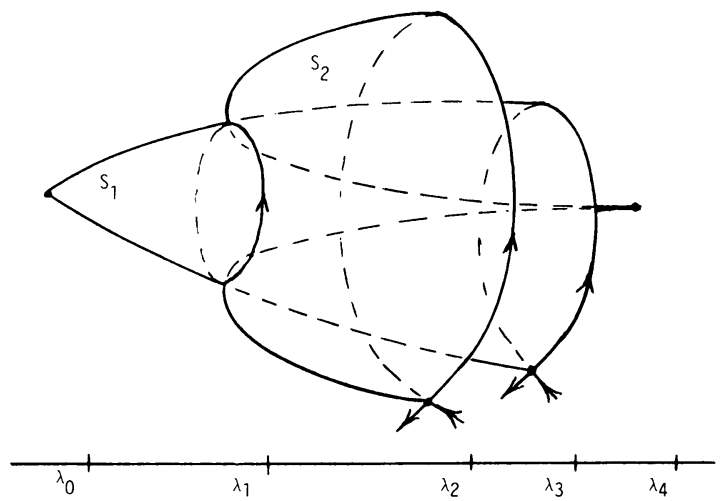

FIgURE 4. A global family of limit cycles $S$ composed of two one-parameter families $S_{1}$ and $S_{2}$ that intersect in a (pitchfork) bifurcation orbit at $\lambda_{1}$ and terminate at Hopf bifurcations at $\lambda_{0}$ and $\lambda_{4}$ and on graphics at $\lambda_{2}$ and $\lambda_{3}$.

the periodic solutions $x(t, \lambda)=(\lambda \cos \lambda t, \lambda \sin \lambda t)$ of the system

$$
\dot{x}=-\lambda y+x\left(r^{2}-\lambda^{2}\right), \quad \dot{y}=\lambda x+y\left(r^{2}-\lambda^{2}\right)
$$

which approach the origin as $\lambda \rightarrow 0$; the origin is a degenerate critical point of this system for $\lambda=0$ since $\operatorname{Df}(0,0)=0$.

Before beginning the local analysis in $\S 1$, it is useful to have a geometric picture of a global family of limit cycles in mind. A global family of limit cycles $S$ is a maximal connected set of limit cycles of $\left(1_{\lambda}\right)$; i.e., geometrically speaking, $S$ is a connected union of two-dimensional surfaces; see Figure 4. Each surface in $S$ represents a maximal one-parameter family of limit cycles, the $\lambda$ cross sections being limit cycles of $\left(1_{\lambda}\right)$ and the surfaces intersecting in bifurcation orbits. In this context, any cyclic family is topologically a twodimensional torus.

\section{Local ANALYSIS}

Consider a relatively prime, planar, analytic system depending on a parameter $\lambda \in \mathbf{R}$ :

$$
\dot{x}=P(x, y, \lambda), \quad \dot{y}=Q(x, y, \lambda) ;
$$


i.e., $P$ and $Q$ do not have a common analytic factor in any neighborhood of any point $(x, y, \lambda) \in \mathbf{R}^{3}$ and $P$ and $Q$ have convergent power series in some neighborhood of every point $(x, y, \lambda) \in \mathbf{R}^{3}$.

Assume that $\left(1_{\lambda}\right)$ has a cycle $L_{0}$ of period $T_{0}$ at the parameter value $\lambda_{0}$ :

$$
L_{0}: \quad \begin{aligned}
& x=\phi_{0}(t), \quad 0 \leq t \leq T_{0} . \\
& y=\psi_{0}(t), \quad .
\end{aligned}
$$

Let $\omega_{0}= \pm 1$ according to whether $L_{0}$ is positively or negatively oriented, respectively.

In order to develop some properties of the Poincare map in a neighborhood of the cycle $L_{0}$, let $l$ be an analytic transversal to the cycle $L_{0}$ through the point $p_{0}=\left(\phi_{0}(0), \psi_{0}(0)\right) \in L_{0}$; i.e.,

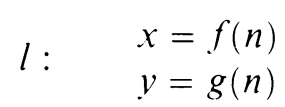

where $f$ and $g$ are analytic for $|n|<\delta, p_{0}=(f(0), g(0))$, and $l$ is not tangent to $L_{0}$ at $p_{0}$; i.e.,

$$
\left|\begin{array}{ll}
\phi_{0}^{\prime}(0) & f^{\prime}(0) \\
\psi_{0}^{\prime}(0) & g^{\prime}(0)
\end{array}\right| \neq 0
$$

The parameter $n$ is taken as the signed arc length along $l, n$ being positive on the exterior of $L_{0}$ and negative on the interior of $L_{0}$. As in [6, p. 110], for a given cycle $L_{0}$ there exists a $\delta>0$ such that for $|n|<\delta$

$$
\begin{aligned}
& l_{s}: \quad x=\phi_{0}(s)+n \omega_{0} \psi_{0}^{\prime}(s) / \sqrt{\phi_{0}^{\prime}(s)^{2}+\psi_{0}^{\prime}(s)^{2}} \\
& y=\psi_{0}(s)-n \omega_{0} \phi_{0}^{\prime}(s) / \sqrt{\phi_{0}^{\prime}(s)^{2}+\psi_{0}^{\prime}(s)^{2}}
\end{aligned}
$$

defines a one-parameter family of nonintersecting analytic transversals which are normal to $L_{0}$ at $p_{s}=\left(\phi_{0}(s), \psi_{0}(s)\right) \in L_{0}$.

Lemma 1.1 (The Poincaré Map). Let $L_{0}$ be a cycle of $\left(1_{0}\right)$ and let $l$ be an analytic transversal through the point $p_{0} \in L_{0}$. Then there exist $a \delta>0$ and unique functions $\tau(n, \lambda)$ and $h(n, \lambda)$, analytic for $|n|<\delta$ and $|\lambda|<\delta$, such that $\tau(0,0)=T_{0}, h(0,0)=0$, and for all $n \in(-\delta, \delta)$ and $\lambda \in(-\delta, \delta)$, the trajectory of $(1$,$) that crosses l$ at the point $(f(n), g(n))$ at time $t=0$ crosses $l$ again at the point $(f(h(n, \lambda)), g(h(n, \lambda)))$ at time $t=\tau(n, \lambda)$; furthermore, for $0<t<\tau(n, \lambda)$, this trajectory has no points in common with $l$.

Remark 1.1. The proof of Lemma 1.1, based on the implicit function theorem for analytic functions in [14], is standard fare; e.g., see [6, 15, 16]. Furthermore, it can be shown that if $L_{0}$ is a cycle of $\left(1_{0}\right)$ and $l_{s}$ is an analytic family of nonintersecting transversals through points $p_{s} \in L_{0}$, such that given by (2), then there exist a $\delta>0$ and unique functions $\tau(s, n, \lambda)$ and $h(s, n, \lambda)$ which are $T_{0}$-periodic in $s$ and analytic for $|s|<\infty,|n|<\delta$, and $|\lambda|<\delta$ such that $\tau(s, 0,0)=T_{0}, h(s, 0,0)=0$ and for all $s,|n|<\delta$, and $|\lambda|<\delta$, the trajectory of $\left(1_{\lambda}\right)$ that crosses $l_{s}$ at a point $(x(s, n), y(s, n))$ at time 
$t=0$ crosses $l_{s}$ again at a point $(x(s, h(s, n, \lambda)), y(s, h(s, n, \lambda)))$ at time $t=\tau(s, n, \lambda)$. If the analytic family of nonintersecting transversals $l_{s}$ is taken as the family of straight lines normal to $L_{0}$, then the functions $x(s, n)$ and $y(s, n)$ are given by $(2)$.

Let $h(n, \lambda)$ be the function defined in Lemma 1.1, let $l(n)$ denote the point $(f(n), g(n))$ on the transversal $l$, and let $\Gamma(\lambda, p)$ denote the trajectory of $\left(1_{\lambda}\right)$ through the point $p$ at time $t=0$. The next corollary relating closed trajectories of $\left(1_{\lambda}\right)$ to zeros of the displacement function, defined below, follows immediately from Lemma 1.1 .

Definition 1.1. The function

$$
d(n, \lambda)=h(n, \lambda)-n
$$

is called the displacement function along the transversal $l$.

Corollary 1.1. The trajectory $\Gamma(\lambda, l(n))$ of $\left(1_{\lambda}\right)$ is closed iff $d(n, \lambda)=0$. If $d(n, \lambda)=0$ then $\tau(n, \lambda)$ is the period of the cycle $L_{\lambda}=\Gamma(\lambda, l(n))$ of $\left(1_{\lambda}\right)$ through the point $l(n)$.

It follows from this corollary that a study of the limit cycles $L_{\lambda}$ of $\left(1_{\lambda}\right)$ in a neighborhood of a given limit cycle $L_{0}$ of $\left(1_{0}\right)$ amounts to a study of the relation $d(n, \lambda)=0$ defined by the displacement function $d(n, \lambda)$ along the transversal $l$. The next lemma is an immediate consequence of the implicit function theorem.

Lemma 1.2. Let $L_{0}$ be a cycle of $\left(1_{0}\right)$ and let $l$ be an analytic transversal to $L_{0}$. Then if $d_{n}(0,0) \neq 0$, there exist $a \delta>0$ and a unique function $n(\lambda)$ such that for all $|\lambda|<\delta, n(\lambda)$ is analytic, $d(n(\lambda), \lambda)=0$, and

$$
n^{\prime}(\lambda)=-\frac{d_{\lambda}(n(\lambda), \lambda)}{d_{n}(n(\lambda), \lambda)} .
$$

In this case it follows that for all $|\lambda|<\delta$ there exists a unique limit cycle $L_{\lambda}$ of $\left(1_{\lambda}\right)$ through each point $l(n(\lambda))$ on the transversal $l$; i.e., $L_{0}$ belongs to a (local) one-parameter family of limit cycles. The next result follows as on pp. 118 and 383 in [6].

Lemma 1.3. Let $d(n, \lambda)$ be the displacement function along a straight line normal to the cycle $L_{0}$. Then

$$
d_{n}(0,0)=\exp \int_{0}^{T_{0}}\left[P_{x}\left(\phi_{0}(t), \psi_{0}(t), 0\right)+Q_{y}\left(\phi_{0}(t), \psi_{0}(t), 0\right)\right] d t-1 .
$$

Definition 1.2. A cycle $L_{0}$ of $\left(1_{0}\right)$ is called a simple limit cycle if

$$
\int_{0}^{T_{0}}\left[P_{x}\left(\phi_{0}(t), \psi_{0}(t), 0\right)+Q_{y}\left(\phi_{0}(t), \psi_{0}(t), 0\right)\right] d t \neq 0 .
$$

If $L_{0}$ is a limit cycle and the above condition is not satisfied, then $L_{0}$ is called a multiple limit cycle. 
Note that bifurcations in a planar family of limit cycles occur only at multiple limit cycles or at a cycle belonging to a continuous band of cycles. The multiplicity of a limit cycle is defined following Andronov et al. [6, p. 272].

Definition 1.3. If $L_{0}$ is a (limit) cycle of $\left(1_{0}\right)$ and if

$$
d_{n}(0,0)=\cdots=d_{n}^{(k-1)}(0,0)=0, \quad d_{n}^{(k)}(0,0) \neq 0
$$

then $L_{0}$ is called a limit cycle of multiplicity $k$.

Remark 1.2. It follows from Remark 1.1 that if $l_{s}$ is an analytic family of nonintersecting transversals to $L_{0}$, it is possible to define a displacement function $d(s, n, \lambda)=h(s, n, \lambda)-n$ along each transversal $l_{s}$. The function $d(s, n, \lambda)$ is $T_{0}$-periodic in $s$ and analytic in a neighborhood of $(s, 0,0)$ for each $s \in \mathbf{R}$. Furthermore, it follows from the periodicity of $\phi_{0}(t)$ and $\psi_{0}(t)$ that for all $s, d_{n}(s, 0,0)$ is given by equation (3); and this in turn implies that if $d_{n}(s, 0,0) \neq 0$ at one particular value of $s$, then $d_{n}(s, 0,0) \neq 0$ for all $s$. In this case, there exist a $\delta>0$ and a unique function $n(s, \lambda)$ which is $T_{0}$-periodic in $s$, analytic in $s$ and $\lambda$, and satisfies $d(s, n(s, \lambda), \lambda)=0$ for all $s$ and $|\lambda|<\delta$.

Remark 1.3. Theorem 42 on p. 277 in [6] states that if $L_{0}$ is a limit cycle of multiplicity $k \geq 2$ of the analytic system $\left(1_{0}\right)$, then there exists an analytic perturbation of $\left(1_{0}\right)$ which has exactly $k$ limit cycles in a neighborhood of $L_{0}$. This shows that the above definition of multiplicity is independent of the transversal along which the displacement function $d(n, \lambda)$ is defined. This is consistent with Francoise and Pugh's definition of multiplicity given on p. 145 in [4]. If the multiplicity $k$ of $L_{0}$ is odd, then $L_{0}$ is either stable or unstable, and if $k$ is even, then $L_{0}$ is a semistable limit cycle. It should also be noted that for an analytic system $d_{n}^{(k)}(0,0)=0$ for $k=1,2,3, \ldots$ if and only if $L_{0}$ is a cycle which belongs to a continuous band of cycles of $\left(1_{0}\right)$. This result does not hold for $C^{\infty}$-systems.

Let us next consider the number of limit cycles that bifurcate from a multiple limit cycle $L_{0}$ of $\left(1_{0}\right)$. It follows from the Weierstrass preparation theorem [6, p. 388] that if $L_{0}$ is a limit cycle of multiplicity $k \geq 2$ then the implicit function $n(\lambda)$ defined by the relation $d(n, \lambda)=0$, where $d(n, \lambda)$ is the displacement function along a transversal $l$, has at most $k$ real branches: $n_{1}(\lambda), n_{2}(\lambda), \ldots, n_{m}(\lambda)$, where $0 \leq m \leq k$. Note that the number of branches, $m$, is independent of the transversal $l$. And each branch, $n_{j}(\lambda)$, defines a local one-parameter family of limit cycles of $\left(1_{\lambda}\right)$. This result also holds when $L_{0}$ is a cycle which belongs to a continuous band of cycles; see Remark 2 at the end of the Appendix. The implicit function $n(\lambda)$ defined by the relation $d(n, \lambda)=0$ is referred to as the limit-cycle function in this paper, since it determines the distance along the transversal $l$ at which $\left(1_{i}\right)$ has a limit cycle.

Definition 1.4. Let $L_{0}$ be a limit cycle of multiplicity $k \geq 2$. If the number of branches $m$ of $d(n, \lambda)=0$ is zero, $L_{0}$ is called an isolated limit cycle. 
An isolated limit cycle is isolated not only from other cycles in the $(x, y)$ plane but also from other cycles of $\left(1_{\lambda}\right)$ in $(x, y, \lambda)$ space; i.e., $L_{0}$ does not belong to any family of limit cycles. For example, the planar system in the introduction with $\Psi(r, \lambda)=\left(r^{2}-1\right)^{2}+\lambda^{2}$ has an isolated limit cycle for $\lambda=0$ which is a circle of radius 1 centered at the origin.

If $L_{0}$ is not an isolated limit cycle, then as in Lemma 1 on p. 396 in [6], for each branch $n(\lambda)$ of the limit cycle function there exists a $\delta>0$ and unique, relatively prime, positive integers $p$ and $q$ such that $n(\lambda)$ can be expanded in a Puiseux series of the form

$$
n(\lambda)=( \pm \lambda)^{p / q} \sum_{j=0}^{\infty} a_{j}( \pm \lambda)^{j / q}
$$

which converges for $0 \leq \pm \lambda<\delta$. Suppose that the first of these two possibilities holds; i.e., the Puiseux series (4) with the positive signs converges for $0 \leq \lambda<\delta$. Let us consider two cases.

Case 1. If $q$ is $o d d$, define $\beta=\lambda^{1 / q}$. It then follows that there exists a $\delta_{1}>0$ such that

$$
n\left(\beta^{q}\right)=\beta^{p} \sum_{j=0}^{\infty} a_{j} \beta^{j}
$$

for $|\beta|<\delta_{1}$ and the analytic continuation of $n\left(\beta^{q}\right)$ from $0 \leq \beta<\delta^{1 / q}$ to $|\beta|<\delta_{1}$ is unique. In this case, the function $n(\lambda)$ defined by its Puiseux series on $0 \leq \lambda<\delta$ is simply continued to a function defined by the same Puiseux series which converges on $|\lambda|<\delta_{1}^{q}$. And this continuation is unique.

Case 2. If $q$ is even, then $p$ is odd and there is a $\delta_{2}>0$ such that the inverse of $n(\lambda)$ exists and satisfies

$$
\lambda(n)=n^{q / p} \sum_{j=0}^{\infty} b_{j} n^{j / p}
$$

for $0 \leq n<\delta_{2}$ if $a_{0}>0$ or for $-\delta_{2}<n \leq 0$ if $a_{0}<0$. Then, as in Case 1 (by introducing $\beta=n^{1 / p}$ ), there is a unique continuation of $\lambda(n)$ (such that $\lambda\left(\beta^{p}\right)$ is analytic for $|\beta|<\delta_{2}^{1 / p}$ ) which is given by the above Puiseux series for $\lambda(n)$ on $|n|<\delta_{2}$. Now let $\lambda=\beta^{q}$ and $\beta= \pm \lambda^{1 / q}$ with $\operatorname{sgn}(\beta)=\operatorname{sgn}(n)$. Then

$$
\beta=n^{1 / p}\left[\sum_{j=0}^{\infty} b_{j} n^{j / p}\right]^{1 / q}
$$

for $|n|<\delta$. Furthermore, since $p$ is odd this function is one-to-one in a neighborhood of $(0,0)$ and there exists a $\delta_{3}>0$ such that its inverse is the unique analytic continuation of the function $n\left(\beta^{q}\right)$, defined on $0 \leq \beta<\delta^{1 / q}$, to the interval $|\beta|<\delta_{3}$; the Taylor series for $n\left(\beta^{q}\right)$ is given by (5) for $|\beta|<\delta_{3}$. 
For example, if $a_{0}>0$, this amounts to continuing the upper branch $n(\lambda)=$ $n_{+}(\lambda)$, given by the Puiseux series (4) on $0 \leq \lambda<\delta$, to the lower branch, given by

$$
n_{-}(\lambda)=\lambda^{p / q} \sum_{j=0}^{\infty}(-1)^{j+1} a_{j} \lambda^{j / q}
$$

on $0 \leq \lambda<\delta_{3}^{1 / q}$. This is the type of continuation that occurs in the case of a jug-handle bifurcation; see Figure $1(\mathbf{a})$.

These results are summarized in the following lemma.

Lemma 1.4. If $L_{0}$ is not an isolated limit cycle, then for each branch $n(\lambda)$ of the limit-cycle function defined by $d(n, \lambda)=0$, where $d(n, \lambda)$ is the displacement function along an analytic transversal $l$ to $L_{0}$, there exist $a \delta>0$ and unique relatively prime positive integers $p$ and $q$ such that $n(\lambda)$ can be expanded in a Puiseux series (4) which converges for $0 \leq \lambda<\delta$ or $-\delta<\lambda \leq 0$; furthermore, there is a $\delta_{1}>0$ such that the function $n\left(\beta^{q}\right)$ has a unique analytic continuation to the interval $|\beta|<\delta_{1}$, given by the Taylor series (5).

Remark 1.4. As in Remark 1.2, each branch $n(s, \lambda)$ defined by $d(s, n, \lambda)=0$ is $T_{0}$-periodic and analytic in $s$, and for each fixed $s \in \mathbf{R}, n(s, \lambda)$ satisfies the conclusions of the above lemma, the integers $p$ and $q$ being independent of $s$.

Let us next introduce the concept of arcs and paths of limit cycles. As in [8], a periodic orbit is regarded as a set in $(\lambda, x, y)$ space; i.e., a periodic orbit of $\left(1_{\lambda}\right)$ is a pair $(\lambda, L)$ where $\lambda \in \mathbf{R}$ and $L$ is a cycle of $\left(1_{\lambda}\right)$.

Definition 1.5. Let $\left(\lambda_{0}, L_{0}\right)$ be a periodic orbit of $\left(1_{\lambda_{0}}\right)$. A mapping

$$
\gamma:\left[\beta_{0}, \beta_{1}\right) \rightarrow \mathbf{R} \times \mathbf{R}^{2}
$$

is called an arc of limit cycles emanating from a point $\left(\lambda_{0}, p_{0}\right) \in\left(\lambda_{0}, L_{0}\right)$ if

(1) $\gamma$ is continuous on $\left[\beta_{0}, \beta_{1}\right)$.

(2) $\gamma\left(\beta_{0}\right)=\left(\lambda_{0}, p_{0}\right)$ and for all $\beta \in\left(\beta_{0}, \beta_{1}\right), \gamma(\beta)=\left(\lambda_{\beta}, p_{\beta}\right)$, a point on a periodic orbit $\left(\lambda_{\beta}, L_{\beta}\right)$ of $\left(1_{\lambda_{\beta}}\right)$ where $L_{\beta}$ is a simple limit cycle of $\left(1_{\lambda_{\beta}}\right)$.

(3) $\gamma_{\beta}$ is a strictly monotone function of $\beta$ on $\left(\beta_{0}, \beta_{1}\right)$.

(4) The period $T_{\beta}$ of $L_{\beta}$ is continuous on $\left[\beta_{0}, \beta_{1}\right)$.

Furthermore, $\gamma$ is called an analytic arc of limit cycles if $\gamma(\beta)$ and $T_{\beta}$ are analytic on $\left(\beta_{0}, \beta_{1}\right)$.

Lemma 1.5. Let $L_{0}$ be a cycle of $\left(1_{i_{0}}\right)$. Each branch $n(\lambda)$ of the limit-cycle function defined by $d(n, \lambda)=0$, where $d(n, \lambda)$ is the displacement function along an analytic transversal $l$ through $p_{0} \in L_{0}$, defines an analytic arc of limit cycles emanating from the point $\left(\lambda_{0}, p_{0}\right)$.

Proof. Let $n(\lambda)$ be a branch of the limit-cycle function defined by $d(n, \lambda)=0$ and suppose that $n(\lambda)$ is defined for $\lambda_{0} \leq \lambda<\lambda_{0}+\delta$. Let $\lambda_{\beta}=\lambda_{0}+\beta \delta$. Then 
for $\beta \in[0,1), p_{\beta}=l\left(n\left(\lambda_{\beta}\right)\right)$ is a point on a limit cycle of $\left(1_{\lambda_{\beta}}\right)$ with period $T_{\beta}=\tau\left(n\left(\lambda_{\beta}\right), \lambda_{\beta}\right)$, given by Lemma 1.1. It is then easy to check that for $\delta>0$ sufficiently small and $\beta \in[0,1), \gamma(\beta)=\left(\lambda_{\beta}, p_{\beta}\right)$ satisfies all of the above requirements for an analytic arc of limit cycles emanating from $\left(\lambda_{0}, p_{0}\right)$.

Definition 1.6. Let $J \subset \mathbf{R}$ be an interval. A mapping

$$
\gamma: J \rightarrow \mathbf{R} \times \mathbf{R}^{2}
$$

is called a path of limit cycles if

(1) $\gamma$ is continuous on $J$,

(2) for all $\beta \in J, \gamma(\beta)=\left(\lambda_{\beta}, p_{\beta}\right)$ is a point on a periodic orbit $\left(\lambda_{\beta}, L_{\beta}\right)$ where, except for possibly a finite number of $\beta$ in any compact subset of $J, L_{\beta}$ is a simple limit cycle of $\left(1_{\lambda_{\beta}}\right)$,

(3) if for $\beta_{1}, \beta_{2} \in J^{0}, \gamma\left(\beta_{1}\right)$ and $\gamma\left(\beta_{2}\right)$ are points on the same periodic orbit, then $\beta_{1}=\beta_{2}$, and

(4) the period $T_{\beta}$ of $L_{\beta}$ is continuous on $J$.

Furthermore, $\gamma$ is called an analytic path of limit cycles if $\gamma(\beta)$ and the period $T_{\beta}$ are analytic on $J^{0}$.

Note that every analytic arc of limit cycles is an analytic path of limit cycles. Also, note that (3) in the above definition implies that a path does not run back and forth across the same set of orbits. $J^{0}$ denotes the interior of $J$ in the above definition. And, as in [8], $\operatorname{dom}(\gamma)$ will denote the domain, $J$, of the path $\gamma$.

For an analytic system $\left(1_{\lambda}\right)$, it can be shown that the periodic orbits $\left(\lambda, L_{\lambda}\right)$, where $L_{\lambda}$ is a multiple limit cycle of $\left(1_{\lambda}\right)$, are isolated in $\mathbf{R} \times \mathbf{R}^{2}$. This fact is used in the proof of the next lemma and in the proof of Theorem 1 below.

Lemma 1.6. Let $L_{0}$ be a cycle of $\left(1_{\lambda_{0}}\right)$. Then for each branch $n(\lambda)$ of the limit-cycle function defined by $d(n, \lambda)=0$, where $d(n, \lambda)$ is the displacement function along an analytic transversal $l$ to $L_{0}$, there exist a $\delta>0$ and a positive integer $q$ such that the mapping

$$
\gamma(\beta)=\left(\lambda_{\beta}, l\left(n\left(\lambda_{\beta}\right)\right)\right)
$$

with $\lambda_{\beta}=\lambda_{0}+\beta^{q}$, defined for $|\beta|<\delta$, is an analytic path of limit cycles.

Proof. If $L_{0}$ is a simple limit cycle, then by Lemma 1.2 there are a $\delta>0$ and a unique function $n(\lambda)$ defined by $d(n, \lambda)=0$ which is analytic for $\left|\lambda-\lambda_{0}\right|<\delta$. Thus, if $\lambda_{\beta}=\lambda_{0}+\beta$ and $p_{\beta}=l\left(n\left(\lambda_{\beta}\right)\right)$, it follows that $\gamma(\beta)=\left(\lambda_{\beta}, p_{\beta}\right)$ is an analytic path of limit cycles with $\gamma:(-\delta, \delta) \rightarrow \mathbf{R} \times \mathbf{R}^{2}$. On the other hand, if $L_{0}$ is a multiple limit cycle, then by Lemma 1.4 there are a $\delta>0$ and a positive integer $q$ such that $n\left(\beta^{q}\right)$ is analytic for $|\beta|<\delta$. Thus, if $\lambda_{\beta}=\lambda_{0}+\beta^{q}$ and $p_{\beta}=l\left(n\left(\lambda_{\beta}\right)\right)$, it follows that $\gamma(\beta)=\left(\lambda_{\beta}, p_{\beta}\right)$ is an analytic path of limit cycles with $\gamma:(-\delta, \delta) \rightarrow \mathbf{R} \times \mathbf{R}^{2}$. In particular, condition (3) in Definition 1.6 
follows from the fact that trajectories of $\left(1_{\lambda}\right)$ do not cross; i.e., if $\gamma\left(\beta_{1}\right)$ and $\gamma\left(\beta_{2}\right)$ were points on the same periodic orbit, it would follow that $\lambda_{\beta_{1}}=\lambda_{\beta_{2}}$ and there is a cycle of the system $\left(1_{\lambda}\right)$ with $\lambda=\lambda_{\beta_{1}}$ that passes through the two points $p_{1}$ and $p_{2}$ on $l$ defined by $p_{\beta}=l\left(n\left(\lambda_{\beta}\right)\right)$ for $\beta=\beta_{1}$ and $\beta=\beta_{2}$, respectively. Now if $q$ is odd, then $\lambda_{\beta}=\lambda_{0}+\beta^{q}$ is $1: 1$ in a neighborhood of $\beta=0$, and $\lambda_{\beta_{1}}=\lambda_{\beta_{2}}$ then implies that $\beta_{1}=\beta_{2}$. And if $q$ is even, then $p$ is odd and the function $n\left(\lambda_{\beta}\right)$, defined by equation (5), is $1: 1$ in a neighborhood of $\beta=0$. Thus, if $\beta_{1} \neq \beta_{2}$ it follows that $p_{1} \neq p_{2}$, and if $p_{1}, p_{2} \in l$ were points on the same cycle $L$ of $\left(1_{\lambda}\right)$, with $\lambda=\lambda_{1}=\lambda_{2}$, it would follow that the planar trajectory $L$ must cross itself, a contradiction. Therefore, $\beta_{1}=\beta_{2}$. And in either case, all of the conditions in Definition 1.6 are met.

If in the above lemma the analytic transversal $l$ is taken as a straight line normal to $L_{0}$, then the path $\gamma$ will be called the analytic path defined by a branch of the limit cycle function $n(\lambda)$ along the normal $l$ to $L_{0}$. The following terminology was introduced in [8].

Definition 1.7. Let $\gamma$ and $\gamma_{0}$ be paths. If for all $\beta_{0} \in \operatorname{dom}\left(\gamma_{0}\right)$ there exists a $\beta \in \operatorname{dom}(\gamma)$ such that $\gamma_{0}\left(\beta_{0}\right)$ and $\gamma(\beta)$ are points on the same periodic orbit, then $\gamma$ is called an extension of $\gamma_{0}$. An extension $\gamma$ of $\gamma_{0}$ is called strict if $\gamma_{0}$ is not an extension of $\gamma$. If a path has no strict extensions, it is called a maximal path. A path $\gamma$ with $\operatorname{dom}(\gamma)=J$ is open at an endpoint $\beta_{0}$ of the interval $J$ if $\beta_{0} \notin J$; it is closed at $\beta_{0}$ if $\beta_{0} \in J$. A path is open if it is open at both endpoints.

Also, as in [8], for a given path of limit cycles $\gamma, \operatorname{Orb}(\gamma)$ denotes the set of all periodic orbits for which there exists a $\beta \in \operatorname{dom}(\gamma)$ such that $\gamma(\beta)$ is a point on that periodic orbit. And $\operatorname{Orb}\left[\gamma\left(\beta_{0}\right)\right]$ will denote the periodic orbit $\left(\lambda_{0}, L_{0}\right)$ through the point $\gamma\left(\beta_{0}\right)$; it follows that

$$
\operatorname{Orb}(\gamma)=\bigcup_{\beta \in \operatorname{dom}(\gamma)} \operatorname{Orb}[\gamma(\beta)]
$$

The following definition of a cyclic path is a slight modification of the definition of a cycle path given in [8]. The necessity for this modification is made clear in the next section.

Definition 1.8. A path $\gamma$ is called a cyclic path if either

1. $\operatorname{dom}(\gamma)=\left[\beta_{0}, \beta_{1}\right]$ and $\gamma\left(\beta_{0}\right)$ and $\gamma\left(\beta_{1}\right)$ are points on the same periodic orbit or

2. $\operatorname{dom}(\gamma)=\left[\beta_{0}, \beta_{1}\right)$ and

$$
\lim _{\beta \rightarrow \beta_{1}^{-}} \gamma(\beta)=\left(\lambda_{0}, p_{0}\right),
$$

a point on $\operatorname{Orb}\left[\gamma\left(\beta_{0}\right)\right]$, or

3. $\operatorname{dom}(\gamma)=\left(\beta_{0}, \beta_{1}\right]$ and

$$
\lim _{\beta \rightarrow \beta_{0}^{+}} \gamma(\beta)=\left(\lambda_{0}, p_{0}\right),
$$

a point on $\operatorname{Orb}\left[\gamma\left(\beta_{1}\right)\right]$. 
A one-parameter family of periodic orbits of the analytic system $\left(1_{\lambda}\right)$ is now defined in such a way that there is no ambiguity as to how to follow a given branch through a bifurcation orbit.

Definition 1.9. A one-parameter family of limit cycles of $\left(1_{\lambda}\right)$ is a subset $S \subset$ $\mathbf{R} \times \mathbf{R}^{2}$ such that

(1) there exists a path of limit cycles $\gamma: J \rightarrow \mathbf{R} \times \mathbf{R}^{2}$ with $S=\operatorname{Orb}(\gamma)$ and

(2) for each $\beta_{0} \in J^{0}$ with $\gamma\left(\beta_{0}\right)=\left(\lambda_{0}, L_{0}\right)$ and $L_{0}$ a multiple limit cycle of $\left(1_{\lambda_{0}}\right)$, there exist a $\delta>0$ and an analytic path $\gamma_{0}$ defined by a branch of the limit-cycle function along a normal $l$ to $L_{0}$ with $\operatorname{dom}\left(\gamma_{0}\right)=(-\delta, \delta)$, and $\gamma_{0}(0) \in\left(\lambda_{0}, L_{0}\right)$ such that $\gamma$ is an extension of $\gamma_{0}$.

In this case $S$ is called a one-parameter family of limit cycles generated by the path $\gamma$.

Lemma 1.7. Let $L_{0}$ be a cycle of $\left(1_{\lambda_{0}}\right)$ and let $\gamma_{1}$ and $\gamma_{2}$ be two analytic paths of limit cycles defined by branches of the limit cycle function along normals $l_{1}$ and $l_{2}$ to $L_{0}$. Let $\operatorname{dom}\left(\gamma_{j}\right)=\left(-\delta_{j}, \delta_{j}\right)$ and assume that $\gamma_{j}(0) \in\left(\lambda_{0}, L_{0}\right)$ for $j=1,2$. Then if $\gamma_{2}$ restricted to $\left(-\delta_{2}, 0\right)$ is an extension of $\gamma_{1}$ restricted to $\left(-\delta_{1}, 0\right)$, it follows that there exists a $\delta>0$ such that $\gamma_{2}$ restricted to $\left(0, \delta_{2}\right)$ is an extension of $\gamma_{1}$ restricted to $(0, \delta)$.

Proof. If $L_{0}$ is a simple limit cycle, then as in Remark 1.2 there are a $\delta>0$ and a unique function $n(s, \lambda)$ which is analytic for all $s$ and $\left|\lambda-\lambda_{0}\right|<\delta$ such that $d(s, n(s, \lambda), \lambda)=0$. If the family of transversals $l$ is the family of normals to $L_{0}$ given by (2), then there exist $s_{1}, s_{2} \in \mathbf{R}$ such that $l_{j}=l_{s_{j}}$ for $j=1,2$ and, assuming $\delta \leq \min \left(\delta_{1}, \delta_{2}\right)$, it follows from Lemma 1.6 and the above hypotheses that

$$
\gamma_{j}(\beta)=\left(\lambda_{\beta}, l_{j}\left(n\left(s_{j}, \lambda_{\beta}\right)\right)\right)
$$

for $j=1,2$ and $|\beta|<\delta$ where $\lambda_{\beta}=\lambda_{0}+\beta$. It thus follows that for $|\beta|<\delta$, $\gamma_{1}(\beta)$ and $\gamma_{2}(\beta)$ lie on the same periodic orbit; i.e., $\gamma_{2}$ restricted to $\left(0, \delta_{2}\right)$ is an extension of $\gamma_{1}$ restricted to $(0, \delta)$. On the other hand, if $L_{0}$ is a multiple limit cycle, then under the above hypotheses it follows from Corollary 1.1, Remark 1.4, and Lemma 1.6 that there exist a $\delta>0$ and a unique branch, $n(s, \lambda)$, of the limit-cycle function defined by $d(s, n, \lambda)=0$ such that $\gamma_{j}(\beta)$ is once again given by the formula $(*)$ for some $s_{j} \in \mathbf{R}, j=1,2$, and $|\beta|<\delta$ where $\lambda_{\beta}=\lambda_{0}+\beta^{q}$ in this case. It again follows that for $|\beta|<\delta, \gamma_{1}(\beta)$ and $\gamma_{2}(\beta)$ lie on the same periodic orbit; i.e., $\gamma_{2}$ restricted to $\left(0, \delta_{2}\right)$ is an extension of $\gamma_{1}$ restricted to $(0, \delta)$.

Remark 1.5. If $S=\operatorname{Orb}(\gamma)$ is a one-parameter family of periodic orbits generated by the path $\gamma$ with $\operatorname{dom}(\gamma)=J$, then geometrically speaking the range $\gamma(J)$ is a path that lies on the two-dimensional surface $S \subset \mathbf{R} \times \mathbf{R}^{2}$. It should 
also be noted that if $\gamma:[0, \infty) \rightarrow \mathbf{R} \times \mathbf{R}^{2}$ is a path of periodic orbits then

$$
\tilde{\gamma}(\beta)=\gamma(\tan \beta \pi / 2)
$$

is also a path of periodic orbits with $\operatorname{dom}(\tilde{\gamma})=[0,1)$.

Theorem 1. Assume that $\left(1_{\dot{\lambda}}\right)$ is a relatively prime, planar, analytic system and let $S_{0}$ be a one-parameter family of limit cycles of $\left(1_{\lambda}\right)$ generated by a path $\gamma_{0}$ with $\operatorname{dom}\left(\gamma_{0}\right)=\left[\beta_{0}, \beta_{1}\right)$. Then either there exist a $\delta>0$ and an extension $\gamma$ of $\gamma_{0}$ with $\operatorname{dom}(\gamma)=\left(\beta_{0}-\delta, \beta_{1}\right)$ and $S=\operatorname{Orb}(\gamma)$ a one-parameter family of limit cycles, or $\gamma_{0}$ is a cyclic path.

Remark 1.6. It can also be shown, as in the proof of this theorem, that if the one-parameter family of limit cycles $S_{0}$ is generated by a path $\gamma_{0}$ with $\operatorname{dom}\left(\gamma_{0}\right)=\left[\beta_{0}, \beta_{1}\right]$, then either there exists a $\delta>0$ such that $\gamma_{0}$ can be extended to a path $\gamma$ with $\operatorname{dom}(\gamma)=\left(\beta_{0}-\delta, \beta_{1}+\delta\right)$ or $\gamma_{0}$ is a cyclic path. Similarly, if $\operatorname{dom}\left(\gamma_{0}\right)=\left(\beta_{0}, \beta_{1}\right]$, then either there exists a $\delta>0$ such that $\gamma_{0}$ can be extended to a path $\gamma$ with $\operatorname{dom}(\gamma)=\left(\beta_{0}, \beta_{1}+\delta\right)$ or $\gamma_{0}$ is a cyclic path.

Proof. Let $\gamma_{0}\left(\beta_{0}\right)=\left(\lambda_{0}, p_{0}\right)$ be a point on the periodic orbit $\left(\lambda_{0}, L_{0}\right)$. Since the periodic orbit $\left(\lambda_{0}, L_{0}\right)$ belongs to the one-parameter family of limit cycles $S_{0}$, it follows that $L_{0}$ is not an isolated limit cycle of $\left(1_{\lambda_{0}}\right)$. According to Corollary 1.1, every local one-parameter family of limit cycles through $L_{0}$ is defined by some branch of the limit-cycle function along the straight line normal $l_{0}$ to $L_{0}$ through $p_{0}$. Let $n(\lambda)$ be that branch that locally defines $S_{0}$ and let $\gamma_{1}(\beta)$ with $\operatorname{dom}\left(\gamma_{1}\right)=\left(-\delta_{1}, \delta_{1}\right)$ and $\delta_{1}>0$ be the analytic path defined by $n(\lambda)$ along the normal $l_{0}$ as in Lemma 1.6. Define

$$
\gamma(\beta)= \begin{cases}\gamma_{0}(\beta) & \text { for } \beta \in\left[\beta_{0}, \beta_{1}\right), \\ \gamma_{1}\left(\beta-\beta_{0}\right) & \text { for } \beta \in\left(\beta_{0}-\delta_{1}, \beta_{0}\right]\end{cases}
$$

and consider the following two cases:

Case 1. Suppose that there exists a $\delta>0$ with $\delta \leq \delta_{1}$ such that for all $\beta \in$ $\left(\beta_{0}-\delta, \beta_{0}\right), \gamma(\beta)$ is not a point on a periodic orbit of $S_{0}$. It then follows that the restriction of $\gamma$ to $\left(\beta_{0}-\delta, \beta_{1}\right)$ is a path which is an extension of the path $\gamma_{0}$. Also, since the periodic orbits $\left(\lambda, L_{\dot{\lambda}}\right)$ with $L_{\dot{\lambda}}$ a multiple limit cycle of $\left(1_{\lambda}\right)$ are isolated in $\mathbf{R} \times \mathbf{R}^{2}$, it follows that $\delta>0$ can be chosen sufficiently small that for all $\beta$ with $0<\left|\beta-\beta_{0}\right|<\delta, \gamma(\beta)$ is a point on a periodic orbit $\left(\lambda_{\beta}, L_{\beta}\right)$ where $L_{\beta}$ is a simple limit cycle of $\left(1_{\lambda}\right)$. It then follows from the fact that $S_{0}$ is a one-parameter family of limit cycles and the above definition of the path $\gamma$ that, according to Definition $1.9, S=\operatorname{Orb}(\gamma)$ is a one-parameter family of limit cycles.

Case 2. Suppose that for all $\delta>0$ with $\delta \leq \delta_{1}$ there exists a $\beta^{*} \in\left(\beta_{0}-\right.$ $\left.\delta, \beta_{0}\right)$ such that $\gamma\left(\beta^{*}\right)$, or equivalently $\gamma_{1}\left(\beta^{*}-\beta_{0}\right)$, lies on some periodic orbit $\left(\lambda^{*}, L^{*}\right)$ of $S_{0}=\operatorname{Orb}\left(\gamma_{0}\right)$. Since the multiple limit cycles of $\left(1_{\lambda}\right)$ are isolated, it follows that $\delta>0$ may be chosen small enough that for all $\beta \in\left(\beta_{0}-\delta, \beta_{0}\right)$, 
$\gamma(\beta)$ lies on a periodic orbit $(\lambda, L)$ where $L$ is a simple limit cycle. Then, in particular, $L^{*}$ is a simple limit cycle; i.e., there is only one family of periodic orbits through $\left(\lambda^{*}, L^{*}\right)$. It then follows from the continuity of $\gamma$ that the periodic orbits through the points of the path $\gamma\left[\left(\beta_{0}-\delta, \beta_{0}\right)\right]$ must coincide with the periodic orbits through the points of the path $\gamma_{0}\left[I_{0}\right]$ for some interval $I_{0}=\left(\tilde{\beta}_{0}, \tilde{\beta}_{1}\right) \subset\left(\beta_{0}, \beta_{1}\right)$. It follows from the continuity of solutions with respect to initial conditions and parameters that

$$
\lim _{\beta \rightarrow \tilde{\beta}_{1}^{-}} \gamma_{0}(\beta)=\left(\lambda_{0}, p_{0}\right),
$$

a point on $\left(\lambda_{0}, L_{0}\right)$. Now if $\tilde{\beta}_{1}<\beta_{1}$, this would contradict the fact that $\gamma_{0}$ is a path of limit cycles. This is easy to see in the case when $L_{0}$ is a simple limit cycle, since in that case there is only one family of limit cycles through $\left(\lambda_{0}, L_{0}\right)$. Thus if $\tilde{\beta}_{1}<\beta_{1}$, it follows from the fact that $\gamma_{0}\left(\tilde{\beta}_{1}\right)$ and $\gamma_{0}\left(\beta_{0}\right)$ both lie on the same periodic orbit, $\left(\lambda_{0}, L_{0}\right)$, that there are $\beta \in\left(\beta_{0}, \tilde{\beta}_{1}\right)$ and a $\tilde{\beta} \in\left(\tilde{\beta}_{1}, \beta_{1}\right)$ such that $\gamma_{0}(\beta)$ and $\gamma_{0}(\tilde{\beta})$ lie on the same periodic orbit. But this contradicts the fact that $\gamma_{0}$ is a path of limit cycles; see Definition 1.6. On the other hand, if $L_{0}$ is a multiple limit cycle, it follows from the fact that $S_{0}=\operatorname{Orb}\left(\gamma_{0}\right)$ is a one-parameter family of limit cycles with $\gamma_{0}\left(\tilde{\beta}_{1}\right) \in\left(\lambda_{0}, L_{0}\right)$ and $\tilde{\beta}_{1}$ an interior point of $\operatorname{dom}\left(\gamma_{0}\right)$ that there is an analytic path $\gamma_{2}$, defined by a branch of the limit-cycle function along a normal to $L_{0}$, with $\operatorname{dom}\left(\gamma_{2}\right)=\left(-\delta_{2}, \delta_{2}\right)$ and $\gamma_{2}(0) \in\left(\lambda_{0}, L_{0}\right)$ such that $\gamma_{0}$ is an extension of $\gamma_{2}$. But

$$
\operatorname{Orb}\left[\left.\gamma_{1}\right|_{(-\delta, 0)}\right]=\operatorname{Orb}\left[\left.\gamma_{0}\right|_{\left(\tilde{\beta}_{0}, \tilde{\beta}_{1}\right)}\right] \text {. }
$$

It therefore follows that, for $\delta>0$ sufficiently small, $\gamma_{2}$ restricted to $(-\delta, 0)$ is an extension of $\gamma_{1}$ restricted to $(-\delta, 0)$, and thus, by Lemma 1.7, it follows that $\gamma_{2}$ restricted to $\left(0, \delta_{2}\right)$ is an extension of $\gamma_{1}$ restricted to $(0, \delta)$. This implies that there are $\beta \in\left(\tilde{\beta}_{0}, \tilde{\beta}_{1}\right)$ and $\tilde{\beta} \in\left(\tilde{\beta}_{1}, \beta_{1}\right)$ such that $\gamma_{0}(\beta)$ and $\gamma_{0}(\tilde{\beta})$ lie on the same periodic orbit. But this contradicts the fact that $\gamma_{0}$ is a path of limit cycles. Thus $\tilde{\beta}_{1}=\beta_{1}$. It can be assumed without loss of generality that $\beta_{1}<\infty$. Otherwise the reparametrization in Remark 1.5 can be used to obtain a path with a finite domain. It then follows, according to Definition 1.8, that $\gamma_{0}$ is a cyclic path. This completes the proof of Theorem 1.

\section{Global analysis}

In this section, global or maximal one-parameter families of limit cycles generated by the relatively prime, planar, analytic system $\left(1_{\lambda}\right)$ are considered. As in [8], if $\gamma$ is a maximal path of limit cycles that generates a one-parameter family of limit cycles $S$, then any path that generates $S$ is maximal. Thus, a one-parameter family of limit cycles $S$ is called maximal if there is a maximal path that generates $S$. Clearly, if $S_{0}$ is a one-parameter family of limit cycles, then there exists a unique maximal one-parameter family of limit cycles containing $S_{0}$. Also, it can be shown that if $\gamma_{1}$ and $\gamma_{2}$ are two paths that generate a maximal one-parameter family of limit cycles, then $\gamma_{1}$ and $\gamma_{2}$ are both 
open paths or neither is and both or neither are cyclic; see [8, p. 438]. Thus, a maximal one-parameter family of limit cycles $S$ is called open or cyclic if $S=\operatorname{Orb}(\gamma)$ and the path $\gamma$ is open or cyclic, respectively. Note that maximal one-parameter families of limit cycles are the main objects of study in this paper, just as snakes, or maximal oriented one-parameter families of limit cycles, were the main objects of study in [8]. First of all, let us prove a result analogous to Theorem 6.3 in [8].

Theorem 2. Assume that $\left(1_{\lambda}\right)$ is a relatively prime, planar, analytic system. Then every maximal, one-parameter family of limit cycles is either open or cyclic.

Proof. Let $S=\operatorname{Orb}(\gamma)$ be a maximal one-parameter family of limit cycles. The proof consists of showing that if $S$ is not open then it is cyclic. Let $\operatorname{dom}(\gamma)=J$ and suppose that $J$ is not open. For example, if $J=\left[\beta_{0}, \beta_{1}\right)$ then it follows from Theorem 1 that either there are a $\delta>0$ and an extension $\tilde{\gamma}$ of $\gamma$ with $\operatorname{dom}(\tilde{\gamma})=\left(\beta_{0}-\delta, \beta_{1}\right)$ or $\gamma$ is a cyclic path. But the first of these two possibilities cannot occur, as this would contradict the hypothesis that $\gamma$ is a maximal path. Thus $\gamma$ is cyclic. The other cases with $J=\left[\beta_{0}, \beta_{1}\right]$ or $J=\left(\beta_{0}, \beta_{1}\right]$ are treated similarly; see Remark 1.6.

This result does not hold in higher dimensions. In particular, for $n \geq 3$ a maximal one-parameter family of periodic orbits which is not cyclic may be closed at an endpoint which corresponds to a period-doubling bifurcation orbit; see the examples on pp. 421 and 423 in [8].

An example of a cyclic family was given by the planar system in the introduction with $\Psi(r, \lambda)=\left(r^{2}-2\right)^{2}+\lambda^{2}-1$. The periodic orbits in the family are circles centered at the origin with radius $r=\left[2 \pm\left(1-\lambda^{2}\right)^{1 / 2}\right]^{1 / 2}$. Jug-handle bifurcations occur at $\lambda= \pm 1$ and $r=2^{1 / 2}$. An analytic path that generates this family is given by $\gamma(\beta)=(\cos \beta, \sqrt{2+\sin \beta}, 0)$ with $0 \leq \beta \leq 2 \pi$.

Note that if $\gamma$ with $\operatorname{dom}(\gamma)=\left[\beta_{0}, \beta_{1}\right]$ is a maximal path which is cyclic, then, according to Definition 1.8, $\gamma_{0}=\gamma$ with $\operatorname{dom}\left(\gamma_{0}\right)=\left[\beta_{0}, \beta_{1}\right)$ is also a maximal cyclic path; i.e., even though $\gamma$ is an extension of $\gamma_{0}$, it is not a strict extension of $\gamma_{0}$ since $\gamma_{0}$ is also an extension of $\gamma$. This follows since for all $\beta \in \operatorname{dom}(\gamma)$ there is a $\tilde{\beta} \in \operatorname{dom}\left(\gamma_{0}\right)$ such that $\gamma(\beta)$ and $\gamma(\tilde{\beta})$ lie on the same periodic orbit; e.g., if $\beta=\beta_{1} \in \operatorname{dom}(\gamma)$ then $\tilde{\beta}=\beta_{0} \in \operatorname{dom}\left(\gamma_{0}\right)$. It follows that if the definition of a cyclic path given in [8] is used rather than Definition 1.8, the path $\gamma_{0}$ would be a maximal path which is neither open nor cyclic. This would contradict Theorem 2 above, and it would also contradict Theorem 6.3 in [8]. For example, the path $\gamma(\beta)=(\cos \beta, \sqrt{2+\sin \beta}, 0)$, given above, is a maximal, oriented path which is cyclic; however, $\gamma_{0}=\gamma$ with $\operatorname{dom}\left(\gamma_{0}\right)=[0,2 \pi)$ is not cyclic according to the definition in [8] and therefore the snake $S=\operatorname{Orb}(\gamma)=\operatorname{Orb}\left(\gamma_{0}\right)$ is neither cyclic nor open. Hence, in order to obtain Theorem 2 above or Theorem 6.3 in [8], it is necessary to use Definition 1.8 of this paper. 
In stating the main result of this paper, it is assumed that the relatively prime, planar, analytic system $\left(1_{\lambda}\right)$ has a cycle $L_{0}$ at some parameter value $\lambda=\lambda_{0}$. If $L_{0}$ is a cycle of $\left(1_{\lambda_{0}}\right)$, then exactly one of the following three possibilities occurs:

(1) $L_{0}$ is an isolated limit cycle of $\left(1_{\lambda_{0}}\right)$.

(2) $L_{0}$ is a cycle that belongs to a continuous band of cycles, but $L_{0}$ does not belong to a one-parameter family of limit cycles of $\left(1_{\lambda}\right)$.

(3) $L_{0}$ is a cycle that belongs to a one-parameter family of limit cycles of $\left(1_{\lambda}\right)$.

In the first case, there is nothing to discuss. In the second case, the nature of the continuous band of cycles containing $L_{0}$ was discussed in [3]; i.e., it was shown in [3] that the inner and outer boundaries of a continuous band of cycles each consist of either a critical point or graphic of $\left(1_{\lambda}\right)$ on the Poincare sphere. A discussion of the third case is the main point of this paper.

The main results of this paper are summarized in the following theorem. Before stating this theorem, it is necessary to give a few more definitions. As was stated in the introduction, a graphic or compound separatrix cycle of $\left(1_{\lambda}\right)$ is a connected union of compatibly oriented separatrix cycles of $\left(1_{\lambda}\right)$; a precise definition of a graphic can be found on p. 518 of [3]. As in [3], $\Gamma(\lambda, p)$ will denote a trajectory of $\left(1_{\lambda}\right)$ through the point $p$ at $t=0, \Gamma(t, \lambda, p)$ will denote the point reached at time $t$ on this trajectory, $\omega[\Gamma(\lambda, p)]$ will denote the $\omega$ limit set of the trajectory $\Gamma(\lambda, p)$, and $\alpha[\Gamma(\lambda, p)]$ will denote the $\alpha$-limit set of the trajectory $\Gamma(\lambda, p)$.

Definition 2.1. Let $S=\operatorname{Orb}(\gamma)$ be a one-parameter family of limit cycles of $\left(1_{\lambda}\right)$ with $\gamma(\beta)=\left(\lambda_{\beta}, p_{\beta}\right)$, a point on a periodic orbit $\left(\lambda_{\beta}, L_{\beta}\right)$ for each $\beta \in \operatorname{dom}(\gamma)$. Let $\beta_{0}$ be an endpoint of $\operatorname{dom}(\gamma)$ with $\lim _{\beta \rightarrow \beta_{0}} \lambda_{\beta}=\lambda_{0}$. Then the limit set of the set of limit cycles $L_{\beta}$ as $\beta \rightarrow \beta_{0}$,

$\lim _{\beta \rightarrow \beta_{0}} L_{\beta}=\left\{p \in \mathbf{R}^{2} \mid\right.$ there exist a sequence $\beta_{n} \rightarrow \beta_{0}$

and a sequence $p_{n} \in L_{\beta_{n}}$ with $p_{n} \rightarrow p$ as $\left.n \rightarrow \infty\right\}$.

Also, if $L_{\beta}=\Gamma\left(\lambda_{\beta}, p_{\beta}\right)$, then the norm of the limit cycle $L_{\beta}$,

$$
\left\|L_{\beta}\right\|=\max _{t}\left\|\Gamma\left(t, \lambda_{\beta}, p_{\beta}\right)\right\|,
$$

where the norm on the right-hand side of this equation is the Euclidean norm on $\mathbf{R}^{2}$.

Theorem 3 (Planar Termination Principle). Assume that $\left(1_{\lambda}\right)$ is a relatively prime, planar, analytic system. It then follows that every one-parameter family of limit cycles of $\left(1_{\lambda}\right)$ is contained in a unique, maximal one-parameter family of limit cycles $S$ and that $S$ is either open or cyclic. If $S=\operatorname{Orb}(\gamma)$ is open at an endpoint $\beta_{0}$ of $\operatorname{dom}(\gamma)$ then either

(1) $\lim _{\beta \rightarrow \beta_{0}}\left|\lambda_{\beta}\right|=\infty$,

(2) $\lim _{\beta \rightarrow \beta_{0}}\left\|L_{\beta}\right\|=\infty$, or 
(3) $\lim _{\beta \rightarrow \beta_{0}} \lambda_{\beta}=\lambda_{0}$ and $\lim _{\beta \rightarrow \beta_{0}} L_{\beta}$ is either a critical point or a graphic of $\left(1_{\lambda_{0}}\right)$.

The first part of this theorem follows from Theorems 1 and 2. The proof of the last part of this theorem parallels the proof of Theorem 2 in [3].

First, as in the corollary on p. 425 of [8], since the Poincare continuation is parametrized by $\lambda$, it follows that on any interval $\left(\beta_{0}, \beta_{1}\right)$ where all of the periodic orbits, $\operatorname{Orb}[\gamma(\beta)]$ with $\beta \in\left(\beta_{0}, \beta_{1}\right)$, are simple, the function $\lambda_{\beta}$ is a strictly monotonic function of $\beta$. Thus, $\lim _{\beta \rightarrow \beta_{0}} \lambda_{\beta}$ exists as a real number or is infinite.

In proving the following sequence of lemmas, it is assumed that $\lim _{\beta \rightarrow \beta_{0}}\left|\lambda_{\beta}\right|$ $<\infty$ and $\lim _{\beta \rightarrow \beta_{0}}\left\|L_{\beta}\right\|<\infty$. In this case let $\lambda_{0}=\lim _{\beta \rightarrow \beta_{0}} \lambda_{\beta}$, let $\left(1_{0}\right)$ denote the system $\left(1_{\lambda}\right)$ with $\lambda=\lambda_{0}$, and let $C_{0}$ denote the limit set $C_{0}=\lim _{\beta \rightarrow \beta_{0}} L_{\beta}$. For a given sequence $\beta_{k} \rightarrow \beta_{0}$ as $k \rightarrow \infty$, let $\lambda_{k}=\lambda_{\beta_{k}}$, let $L_{k}$ denote $L_{\beta}$ with $\beta=\beta_{k}$, and let $\left(1_{k}\right)$ denote the system $\left(1_{\lambda}\right)$ with $\lambda=\lambda_{k}$. The first lemma follows as in [3] or as on p. 390 of [15].

Lemma 2.1. $C_{0}=\lim _{\beta \rightarrow \beta_{0}} L_{\beta}$ is a nonempty, closed, connected set.

Lemma 2.2. If $p_{0} \in C_{0}$, then $\Gamma\left(\lambda_{0}, p_{0}\right) \subset C_{0}$.

Proof. If $p \in C_{0}$ is a critical point of $\left(1_{0}\right)$, then $\Gamma\left(\lambda_{0}, p\right)=\{p\} \subset C_{0}$. Otherwise, it follows from the continuity of solutions with respect to initial conditions that if $p_{k} \rightarrow p$ as $k \rightarrow \infty$, then for fixed $t$

$$
\lim _{k \rightarrow \infty} \Gamma\left(t, \lambda_{0}, p_{k}\right)=\Gamma\left(t, \lambda_{0}, p\right) \text {. }
$$

And by Theorem 4.3, p. 59 in [15], it follows that since $\beta_{k} \rightarrow \beta_{0}$ and $\lambda_{k} \rightarrow \lambda_{0}$ as $k \rightarrow \infty$,

$$
\lim _{k \rightarrow \infty} \Gamma\left(t, \lambda_{k}, p_{n}\right)=\Gamma\left(t, \lambda_{0}, p_{n}\right)
$$

uniformly with respect to the $p_{n}$. Then by Moore's theorem [17, p. 100], it follows that for fixed $t$

$$
\lim _{k \rightarrow \infty} \Gamma\left(t, \lambda_{k}, p_{k}\right)=\Gamma\left(t, \lambda_{0}, p\right) .
$$

But if $p_{k} \in L_{k}$, then for each $t \in \mathbf{R}, \Gamma\left(t, \lambda_{k}, p_{k}\right) \in L_{k}$ since $L_{k}$ is a limit cycle of $\left(1_{k}\right)$. It then follows from the definition of the limit set $C_{0}$ that for each $t \in \mathbf{R}, \Gamma\left(t, \lambda_{0}, p\right) \in C_{0}$; i.e., $\Gamma\left(\lambda_{0}, p\right) \subset C_{0}$.

Since Lemma 2.2 implies that $C_{0}$ is an invariant set of $\left(1_{0}\right)$, the next lemma follows from the proposition on p. 240 in [16].

Lemma 2.3. If $p \in C_{0}$ then $\omega\left[\Gamma\left(\lambda_{0}, p\right)\right] \subset C_{0}$ and $\alpha\left[\Gamma\left(\lambda_{0}, p\right)\right] \subset C_{0}$.

Lemma 2.4. If for a given $p \in C_{0}, \omega\left[\Gamma\left(\lambda_{0}, p\right)\right]$ is a cycle of $\left(1_{0}\right)$, it follow's that

$$
\omega\left[\Gamma\left(\lambda_{0}, p\right)\right]=C_{0}=\Gamma\left(\lambda_{0}, p\right) .
$$

Proof. By Lemma 2.3 , for $p \in C_{0}, \omega\left[\Gamma\left(\lambda_{0}, p\right)\right] \subset C_{0}$. It remains to show that if $\omega\left[\Gamma\left(\lambda_{0}, P\right)\right]$ is a cycle of $\left(1_{0}\right)$ then $\omega\left[\Gamma\left(\lambda_{0}, p\right)\right] \subset C_{0}$. In order to prove 
this, it is first necessary to show that if $\omega\left[\Gamma\left(\lambda_{0}, p\right)\right]$ is a cycle, say $L_{0}$, of $\left(1_{0}\right)$, then for any sequence $\beta_{k} \rightarrow \beta_{0}$ and for any sequence of points $q_{k} \in L_{k}$ with $q_{k} \rightarrow q_{0} \in L_{0}$ as $k \rightarrow \infty$, the periods of the cycles $L_{k}$ are uniformly bounded by $T_{0}+1$ for all sufficiently large $k$, where $T_{0}$ is the period of $L_{0}$. To prove this, let $l_{0}$ be the transversal normal to $L_{0}$ at $q_{0}$. Since $q_{k} \rightarrow q_{0} \in L_{0} \cap l$ and since $l_{0}$ is normal to $L_{0}$ at $q_{0}$, it follows from the continuity of the functions $P$ and $Q$ in $\left(1_{\lambda}\right)$ that $l_{0}$ is transverse to $L_{k}$ for all sufficiently large $k$. Hence, by Lemma 12 in [18], it follows that there exist a $\delta>0$ and an integer $K$ such that for all $k \geq K, L_{k}$ crosses the transversal $l_{0}$ only once in a $\delta$-neighborhood of the point $q_{0}$. Let $\tilde{q}_{k}$ be the point of intersection of $L_{k}$ with $l_{0}$. And let $n_{k}$ be the signed distance, positive on the exterior of $L_{0}$ and negative on the interior of $L_{0}$, measured along $l_{0}$ from $q_{0}$ to $\tilde{q}_{k}$. Then if $d(n, \lambda)$ is the displacement function along the transversal $l_{0}$ normal to $L_{0}$ at $q_{0}$, it follows by Corollary 1.1 that $d\left(n_{k}, \lambda_{k}\right)=0$. By Lemma 1.5, each branch $n(\lambda)$ of the limit-cycle function defined by $d(n, \lambda)=0$ defines an analytic arc of limit cycles emanating from the point $\left(\lambda_{0}, q_{0}\right) \in\left(\lambda_{0}, L_{0}\right)$. Since the periodic orbits $\left(\lambda_{k}, L_{k}\right)$ belong to the one-parameter family of limit cycles $S$, the points $\left(n_{k}, \lambda_{k}\right)$ all lie on the same branch; i.e., there exist a $\delta>0$ and a unique function $n(\lambda)$ satisfying $d(n(\lambda), \lambda)=0$ for $\lambda_{0} \leq \lambda<\lambda_{0}+\delta$ such that $n\left(\lambda_{k}\right)=n_{k}$. The periods of the limit cycles $L_{k}$, according to Lemma 1.1, are thus given by $T_{k}=\tau\left(n\left(\lambda_{k}\right), \lambda_{k}\right)$. Hence, if $T_{0}$ is the period of $L_{0}$, it follows from the continuity of $\tau(n, \lambda)$ in Lemma 1.1 and $n(\lambda)$ as in equation (4) that $\lim _{k \rightarrow \infty} T_{k}=T_{0}$. Thus, for all sufficiently large $k$, the periods $T_{k}$ are uniformly bounded by $T_{0}+1$.

Now for any point $p_{0} \in C_{0}$, there exist a sequence $\beta_{k} \rightarrow \beta_{0}$ and a sequence of points $p_{k} \in L_{k}$ such that $p_{k} \rightarrow p_{0}$ as $k \rightarrow \infty$. And if, for $p \in C_{0}$, $\omega\left[\Gamma\left(\lambda_{0}, p\right)\right]$ is a cycle $L_{0}$, it follows from above that for any given sequence $q_{k} \in L_{k}$ with $q_{k} \rightarrow q_{0} \in L_{0}$ as $k \rightarrow \infty$ there is a bounded sequence $t_{k} \in$ $\left[0, T_{0}+1\right]$ such that $p_{k}=\Gamma\left(t_{k}, \lambda_{k}, q_{k}\right)$. Then by continuity of solutions with respect to initial conditions and parameters, uniformly with respect to $t$ on $\left[0, T_{0}+1\right]($ see $[15$, Theorem 4.3, p. 59]), it follows that for all $\varepsilon>0$ there is an integer $N$ such that for all $k \geq N$,

$$
\left\|p_{k}-\Gamma\left(t_{k}, \lambda_{0}, q_{0}\right)\right\|<\varepsilon
$$

Thus,

$$
\lim _{k \rightarrow \infty} \Gamma\left(t_{k}, \lambda_{0}, q_{q}\right)=p_{0}
$$

And since $\Gamma\left(t_{k}, \lambda_{0}, q_{0}\right) \in \omega\left[\Gamma\left(\lambda_{0}, p\right)\right]$ when $\omega\left[\Gamma\left(\lambda_{0}, p\right)\right]$ is a cycle, it follows from the fact that $\omega\left[\Gamma\left(\lambda_{0}, p\right)\right]$ is a closed set (see $[15$, p. 390]) that $p \in \omega\left[\Gamma\left(\lambda_{0}, p\right)\right]$. Hence, since any point $p_{0} \in C_{0}$ belongs to $\omega\left[\Gamma\left(\lambda_{0}, p\right)\right]$, it follows that $C_{0} \subset \omega\left[\Gamma\left(\lambda_{0}, p\right)\right]$. Thus, $C_{0}=\omega\left[\Gamma\left(\lambda_{0}, p\right)\right]$.

Finally, it follows from Lemma 2.3 and the above result that $\Gamma\left(\lambda_{0}, p\right) \subset C_{0}=$ $\omega\left[\Gamma\left(\lambda_{0}, p\right)\right]$. Therefore by Lemmas 2.3 and 2.4 on p. 393 in [15], it follows that 
$\Gamma\left(\lambda_{0}, p\right)=\omega\left[\Gamma\left(\lambda_{0}, p\right)\right]$ if $\omega\left[\Gamma\left(\lambda_{0}, p\right)\right]$ is a cycle. This completes the proof of Lemma 2.4.

Corollary 2.1. For $p \in C_{0}, \omega\left[\Gamma\left(\lambda_{0}, p\right)\right]$ is not a cycle of $\left(1_{0}\right)$.

Proof. If $\omega\left[\Gamma\left(\lambda_{0}, p\right)\right]$ were a cycle, $L_{0}$, then by Lemma $2.4 C_{0}=L_{0}$. But then for $\gamma(\beta)=\left(\lambda_{\beta}, p_{\beta}\right)$, it follows that $\lim _{\beta \rightarrow \beta_{0}} p_{\beta}=p_{0} \in L_{0}$ and $\left(\lambda_{0}, L_{0}\right)$ is a periodic orbit. Thus, either there is a strict extension of $\gamma$ beyond $\beta_{0}$ or $\gamma$ is cyclic. The first of these possibilities is ruled out by the fact that $\gamma$ is a maximal path and the second is ruled out by the hypothesis that $\gamma$ is open. Therefore $\omega\left[\Gamma\left(\lambda_{0}, p\right)\right]$ is not a cycle of $\left(1_{0}\right)$.

Corollary 2.2. For $p \in C_{0}, \Gamma\left(\lambda_{0}, p\right)$ is not a cycle of $\left(1_{0}\right)$.

Proof. If $\Gamma\left(\lambda_{0}, P\right)$ is a cycle then $\omega\left[\Gamma\left(\lambda_{0}, P\right)\right]=\Gamma\left(\lambda_{0}, P\right)$ (see [15]), and therefore $\omega\left[\Gamma\left(\lambda_{0}, P\right)\right]$ is a cycle of $\left(1_{0}\right)$. But this contradicts Corollary 2.1. Thus, $\Gamma\left(\lambda_{0}, P\right)$ is not a cycle of $\left(1_{0}\right)$.

The structure of the limit set $C_{0}$ may be quite complicated, as the oneparameter family of limit cycles $S$ could oscillate down to a rather large complicated set as $\beta \rightarrow \beta_{0}$; however, Lemma 2.4 and its corollaries already establish the fact that $C_{0}$ cannot contain any cycles or limit cycles of $\left(1_{0}\right)$. It is still conceivable that $C_{0}$ could consist of a set consisting of nested graphics which are connected by trajectories which spiral from one graphic to another. The remaining lemmas rule this possibility out and establish that $C_{0}$, in fact, consists of a single graphic or critical point of $\left(1_{0}\right)$.

Lemma 2.5. $C_{0}$ contains at least one and at most a finite number of critical points of $\left(1_{0}\right)$.

Proof. Since $\lim _{\beta \rightarrow \beta_{0}}\left\|L_{\beta}\right\|<\infty$, it follows that there is a $\delta>0$ such that the set of limit cycles $L_{\beta}$, with $\beta \in \operatorname{dom}(\gamma)$ and $\left|\beta-\beta_{0}\right|<\delta$, is contained in a bounded set $\Omega$ of $\mathbf{R}^{2}$. It follows that the limit set $C_{0} \subset \bar{\Omega}$. Thus by Lemma 2.3, for $p \in C_{0}, \omega\left[\Gamma\left(\lambda_{0}, p\right)\right] \subset \bar{\Omega}$. And, according to the Poincaré-Bendixson theorem, $\omega\left[\Gamma\left(\lambda_{0}, p\right)\right]$ either contains a critical point of $\left(1_{0}\right)$ or it is a cycle of $\left(1_{0}\right)$. However, according to Corollary $2.1, \omega\left[\Gamma\left(\lambda_{0}, p\right)\right]$ is not a cycle of $\left(1_{0}\right)$. Thus $\omega\left[\Gamma\left(\lambda_{0}, p\right)\right]$ contains at least one critical point of $\left(1_{0}\right)$. And since, by Lemma $2.3, \omega\left[\Gamma\left(\lambda_{0}, p\right)\right] \subset C_{0}$, it follows that $C_{0}$ contains at least one critical point of $\left(1_{0}\right)$. By Theorem 1 in [3], the relatively prime, analytic system $\left(1_{0}\right)$ has at most a finite number of critical points in $\bar{\Omega}$, and since $C_{0} \subset \bar{\Omega}$ it follows that $C_{0}$ contains at most a finite number of critical points of $\left(1_{0}\right)$.

Lemma 2.6. Either $C_{0}$ is a single critical point of $\left(1_{0}\right)$ or $C_{0}$ is a connected set composed of a finite number of distinct critical points of $\left(1_{0}\right), p_{i}, i=1, \ldots, n$, and a finite number of distinct separatrices of $\left(1_{0}\right), \Gamma_{i}, i=1, \ldots, m$, with $m \geq n$ such that for each $i=1, \ldots, m$, there are integers $j, k \in\{1, \ldots, n\}$ with $\omega\left[\Gamma_{i}\right]=p_{j}$ and $\alpha\left[\Gamma_{i}\right]=p_{k}$, and such that for each $i=1, \ldots, n$ there are integers $j, k \in\{1, \ldots, m\}$ such that $p_{i}=\omega\left[\Gamma_{j}\right]$ and $p_{i}=\omega\left[\Gamma_{k}\right]$; furthermore, 
there is a $\delta>0$ such that for all $\beta \in \operatorname{dom}(\gamma)$ with $\left|\beta-\beta_{0}\right|<\delta$, all of the separatrix cycles contained in $C_{0}$ have the same orientation with respect to some point $p_{0} \in \mathbf{R}^{2}$, as do the limit cycles $L_{\beta}$ of $\left(1_{\beta}\right)$.

Proof. Given $p_{0} \in C_{0}$, if $p_{0}$ is not a critical point of $\left(1_{0}\right)$ then, as in the proof of Lemma 2.5, $\omega\left[\Gamma\left(\lambda_{0}, p_{0}\right)\right]$ contains a critical point of $\left(1_{0}\right)$. Thus, $\Gamma\left(\lambda_{0}, p_{0}\right)$ is not a cycle and by the Poincaré-Bendixson theorem $\omega\left[\Gamma\left(\lambda_{0}, p_{0}\right)\right]$ is either a single critical point or a graphic of $\left(1_{0}\right)$. Let us first show that $\omega\left[\Gamma\left(\lambda_{0}, p_{0}\right)\right]$ is not a graphic of $\left(1_{0}\right)$. If it were a graphic of $\left(1_{0}\right)$ then, as in $[15$, p. 396], the trajectory $\Gamma\left(\lambda_{0}, p_{0}\right)$ spirals out (or down) to $\omega\left[\Gamma\left(\lambda_{0}, p_{0}\right)\right]$. Let $l$ be an analytic transversal through $p_{0}$. Then since $\Gamma\left(\lambda_{0}, p_{0}\right)$ is not a cycle, it crosses $l$ at two distinct points $p_{0}$ and $p_{1}$ on $l$ and nowhere in between. It then follows as in Lemma 13 in [18] that there exist a $\delta>0$ and a displacement function $d(n, \lambda)$ defined and analytic for $|n|<\delta$ and $\left|\lambda-\lambda_{0}\right|<\delta$. Then since $p_{0} \in C_{0}$, there are a sequence $\beta_{k} \rightarrow \beta_{0}$ and a sequence of points $p_{k} \in L_{k}$ such that $p_{k} \rightarrow p_{0}$ as $k \rightarrow \infty$. For all sufficiently large $k$, the points $p_{k}$ may be assumed to lie on the transversal $l$. Otherwise, another transversal could be constructed through these points. Suppose that $p_{k}=l\left(n_{k}\right)$. Then, since $p_{0}=l(0)$, it follows that $n_{k} \rightarrow 0$ as $k \rightarrow \infty$. But it then follows that $d\left(0, \lambda_{0}\right) \neq 0$ since $\Gamma\left(\lambda_{0}, p_{0}\right)$ is not a cycle; but $d\left(n_{k}, \lambda_{k}\right)=0$ since for each $k, L_{k}$ is a cycle. This leads to the following contradiction since $d(n, \lambda)$ is continuous:

$$
0=\lim _{k \rightarrow \infty} d\left(n_{k}, \lambda_{k}\right)=d\left(0, \lambda_{0}\right) \neq 0 .
$$

Thus, $\omega\left[\Gamma\left(\lambda_{0}, p_{0}\right)\right]$ is not a graphic of $\left(1_{0}\right)$; i.e., it is a single critical point of $\left(1_{0}\right)$. It can similarly be shown that $\alpha\left[\Gamma\left(\lambda_{0}, p_{0}\right)\right]$ consists of a single critical point of $\left(1_{0}\right)$.

Next, assume that $C_{0}$ is not a single critical point of $\left(1_{0}\right)$. Then, since, by Lemma 2.1, $C_{0}$ is connected and since the critical points of $\left(1_{0}\right)$ are isolated, it follows that each critical point in $C_{0}$ is either the $\alpha$ or the $\omega$ limit set of some trajectory of $\left(1_{0}\right)$ which, by Lemma 2.2 , is entirely contained in $C_{0}$. Furthermore, it follows from the continuity of solutions with respect to initial conditions and parameters that each critical point is the $\alpha$-limit set of one trajectory in $C_{0}$ and the $\omega$-limit set of another trajectory in $C_{0}$ and that there is a hyperbolic sector at $p_{0}$; i.e., each trajectory in $C_{0}$ is a separatrix of $\left(1_{0}\right)$. But by Bendixson's Theorem IX in [19], there is at most a finite number of separatrices at each critical point of $\left(1_{0}\right)$. And by Theorem 1 in [3], $\left(1_{0}\right)$ has at most a finite number of critical points. Thus there is at most a finite number of critical points $p_{i}, i=1, \ldots, n$, and separatrices $\Gamma_{i}, i=1, \ldots, m$, in $C_{0}$. Clearly, $m \geq n$.

Finally, if $C_{0}$ is not a single critical point of $\left(1_{0}\right)$, it follows from the definition of the limit set $C_{0}$ and the continuity of solutions with respect to initial conditions and parameters that there is a $\delta>0$ such that for all $\beta \in \operatorname{dom}(\gamma)$ with $\left|\beta-\beta_{0}\right|<\delta$, each separatrix cycle in $C_{0}$ has the same orientation as $L_{\beta}$ with respect to some point $p_{0}$ with $p_{0} \in \operatorname{Int}\left(L_{\beta}\right)$ or $p_{0} \in \operatorname{Ext}\left(L_{\beta}\right)$ for all $\beta \in \operatorname{dom}(\gamma)$ with $\left|\beta-\beta_{0}\right|<\delta$. This completes the proof of Lemma 2.6. 
The planar termination principle then follows from Lemma 2.6 and the definition of a graphic given in [3] since, by Lemma 2.6, either $C_{0}$ consists of a single critical point of $\left(1_{0}\right)$ or $C_{0}$ is a loop formed by a finite number of critical points and separatrices connecting them,

$$
p_{1}, \Gamma_{1}, p_{2}, \Gamma_{2}, \ldots, p_{m}, \Gamma_{m}, p_{m+1}=p_{1}
$$

such that, with an appropriate renumbering of the critical points and separatrices in Lemma 2.6,

$$
\begin{gathered}
p_{1}=\alpha\left(\Gamma_{1}\right), \\
\omega\left(\Gamma_{1}\right)=p_{2}=\alpha\left(\Gamma_{2}\right), \ldots, \omega\left(\Gamma_{m-1}\right)=p_{m}=\alpha\left(\Gamma_{m}\right), \\
\omega\left(\Gamma_{m}\right)=p_{m+1}=p_{1} .
\end{gathered}
$$

The fact that the normal orientations of the separatrices in $C_{0}$ are compatible follows from the last statement in Lemma 2.6.

A one-parameter family of limit cycles $S=\operatorname{Orb}(\gamma)$ is called bounded if $\lim _{\beta \rightarrow \beta_{0}}\left|\lambda_{\beta}\right|<\infty$ and $\lim _{\beta \rightarrow \beta_{0}}\left\|L_{\beta}\right\|<\infty$ at both endpoints of $\operatorname{dom}(\gamma)$. Since the periods $T_{\beta}$ remain bounded as a one-parameter family of limit cycles approaches a nondegenerate critical point of $(1 ;)$, the following corollary is an immediate consequence of the planar termination principle:

Corollary. Under the hypotheses of the above theorem, the periods $T_{\beta}$ of a bounded, maximal, one-parameter family of limit cycles $S=\operatorname{Orb}(\gamma)$ become unbounded as $\beta \rightarrow \beta_{0}$, an endpoint of $\operatorname{dom}(\gamma)$, only if $\lim _{\beta \rightarrow \beta_{0}} L_{\beta}$ is either a degenerate critical point or a graphic of $\left(1_{0}\right)$.

\section{EXAMPLES}

An example of a cyclic family and several examples of families of limit cycles with various types of bifurcations were given in the introduction. This section contains examples of bounded and unbounded, open, maximal one-parameter family of limit cycles generated by polynomial systems in the plane.

It was shown in [20] that the quadratic system

$$
\dot{x}=y+y^{2}, \quad \dot{y}=-a x+\lambda y-x y+(\lambda+1) y^{2}
$$

with $a=2$ generates a bounded, open, maximal, one-parameter family of limit cycles with $0<\lambda<\lambda_{0}$ where $\lambda_{0} \cong .52$. See [20, Theorem 3, p. 156, and Figures 9 and 10]. This maximal one-parameter family of limit cycles originates at a Hopf bifurcation at $\lambda=0$ and terminates on a graphic consisting of a simple separatrix loop at a hyperbolic saddle at $\lambda=\lambda_{0}$. Geometrically, the family is a two-dimensional surface in $\mathbf{R}^{3}$ similar to the surface $S_{1}$ shown in Figure 4 of this paper.

If $a=1$, then the above quadratic system generates an unbounded, open, maximal, one-parameter family of limit cycles with $0<\lambda<\lambda_{0}$ where $\lambda_{0} \cong$ 1.57 ; see $[20,21]$. This family also originates at a Hopf bifurcation at $\lambda=0$ 
and terminates on a graphic consisting of two critical points and two separatrices, one of which lies on the equator of the Poincare sphere; see [20, Figure 7, p. 153]. In this context, it can be shown that the planar termination principle established in this paper can be extended to a termination principle for unbounded families where $\left\|L_{\beta}\right\| \rightarrow \infty$ as $\beta \rightarrow \beta_{0}$; i.e., if $\lambda_{\beta} \rightarrow \lambda_{0}$ as $\beta \rightarrow \beta_{0}$ then it can be shown that $\lim _{\beta \rightarrow \beta_{0}} L_{\beta}$ is either a critical point, a cycle, or a graphic of $\left(1_{0}\right)$ on the Poincare sphere provided that the equator of the Poincare sphere does not consist of critical points. Furthermore, if the limit set, $\lim _{\beta \rightarrow \beta_{0}} L_{\beta}$, is a cycle, it consists of the entire equator of the Poincare sphere and the family of limit cycles can be continued through this cycle. Thus, we have the following result for relatively prime polynomial systems; cf. [22]:

Extended planar termination principle: Every one-parameter family of limit cycles of a relatively prime, polynomial system $\left(1_{\lambda}\right)$ is contained in a maximal one-parameter family $S$ which is either open or cyclic. If $S=\operatorname{Orb}(\gamma)$ is open at an endpoint $\beta_{0}$ of $\operatorname{dom}(\gamma)$, then either $\left|\lambda_{\beta}\right| \rightarrow \infty$ as $\beta \rightarrow \beta_{0}$ or $\lambda_{\beta} \rightarrow \lambda_{0}$ as $\beta \rightarrow \beta_{0}$ and the limit set

$$
C_{0}=\lim _{\beta \rightarrow \beta_{0}} L_{\beta}
$$

is either a critical point or a graphic of $\left(1_{\hat{\lambda}_{0}}\right)$ on the Poincare sphere or $C_{0}$ contains an arc of critical points on the equator of the Poincare sphere.

The final example of this paper illustrates the twisting type of behavior that can be exhibited by a "snake," as defined in [8], in contrast to the monotonic

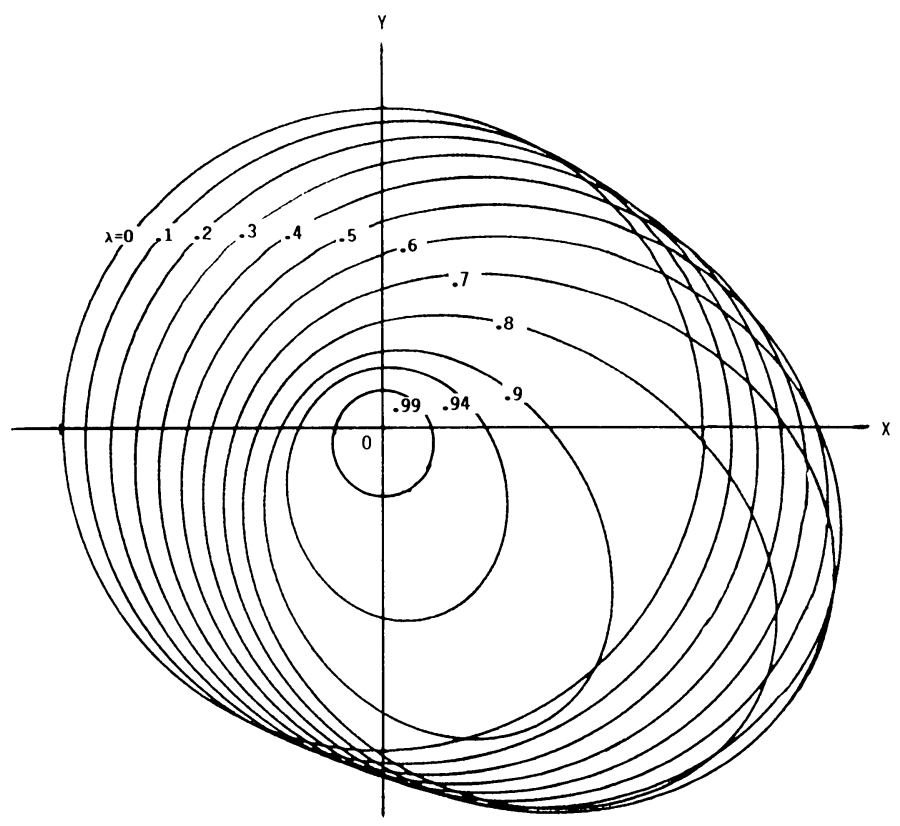

Figure 5 
type of behavior exhibited by any one-parameter family of limit cycles generated by a rotated family of vector fields, described in the appendix at the end of this paper. The one-parameter family of limit cycles generated by the system

$$
\begin{aligned}
& \dot{x}=-y+x\left[(x-\lambda)^{2}+y^{2}-1\right], \\
& \dot{y}=x+y\left[(x-\lambda)^{2}+y^{2}-1\right]
\end{aligned}
$$

has been studied numerically by the author. The family originates at a Hopf bifurcation at the critical point at the origin when $\lambda=-1$ and terminates at the critical point at the origin when $\lambda=1$. Various limit cycles in the family are shown in Figure 5 for $0 \leq \lambda<1$. The corresponding limit cycles in the family for $-1<\lambda \leq 0$ can be obtained by reflecting those in Figure 5 about the origin.

\section{APPENDIX}

The purpose of this appendix is to generalize some of the results for oneparameter families of limit cycles generated by families of rotated vector fields, obtained by Duff [1] and the author [7], and to compare some of those results with the results contained in this paper.

First, the planar family of vector fields described by $\left(1_{\lambda}\right)$ is called a family of rotated vector fields if the critical points of $\left(1_{\lambda}\right)$ remain fixed as $\lambda$ varies in $(-\infty, \infty)$ and if the angle of each vector in the vector field, $\theta=\tan ^{-1} Q / P$, varies monotonically with $\lambda$, i.e., if

$$
\partial \theta / \partial \lambda=\left(P Q_{\lambda}-Q P_{\lambda}\right) /\left(P^{2}+Q^{2}\right)
$$

is not zero for $\lambda \in \mathbf{R}$ or, equivalently, if the determinant

$$
D=\left|\begin{array}{ll}
P & Q \\
P_{\lambda} & Q_{\lambda}
\end{array}\right|
$$

is not zero for $\lambda \in \mathbf{R}$.

The main results of Duff [1] show that the limit cycles in any one-parameter family of limit cycles generated by a family of rotated vector fields $\left(1_{i}\right)$ expand or contract monotonically with $\lambda$ until they intersect one of the critical points of $\left(1_{\lambda}\right)$. The planar termination principle established in this paper generalizes this result due to Duff [1]. The same results were also shown to hold in case the determinant $D$ does not change sign and has only a finite number of zeros on any limit cycle of $\left(1_{\hat{\lambda}}\right)$; see [7].

The following formula, similar to equation (3) in this paper, has been obtained by Andronov et al. [6; cf. equation (36), p. 384]:

$$
d_{\dot{\lambda}}(0,0)=-\omega_{0} I_{0} /\left[P^{2}\left(\phi_{0}(0), \psi_{0}(0), 0\right)+Q^{2}\left(\phi_{0}(0), \psi_{0}(0), 0\right)\right]^{1 / 2}
$$

where the integral

$I_{0}=\int_{0}^{T} \exp \int_{t}^{T}\left[P_{x}\left(\phi_{0}(s), \psi_{0}(s), 0\right)\right.$

$$
\left.+Q_{y}\left(\phi_{0}(s), \psi_{0}(s), 0\right)\right] d s D\left(\phi_{0}(t), \psi_{0}(t), 0\right) d t .
$$


In this equation, $d(n, \lambda)$ denotes the displacement function along the straight line normal $l_{0}$ to $L_{0}$ at the point $p_{0}=\left(\phi_{0}(0), \psi_{0}(0)\right) \in L_{0}, D(x, y, \lambda)$ is the determinant defined above, and $\omega_{0}= \pm 1$ according to the orientation of $L_{0}$. This formula for $d_{\lambda}(0,0)$ also follows from equation (3.17) on p. 23 in [1].

Remark 1. If $l_{s}$ is the straight line normal to $L_{0}$ given by equation (2) in this paper and if $d(s, n, \lambda)$ denotes the displacement function along $l_{s}$ as defined in Remark 1.2, then it can be shown that $d_{\lambda}(s, 0,0)$ is given by the above formula where $I_{0}$ is replaced by an integral $I_{s}$ with the integration running from $s$ to $s+T$ rather than from 0 to $T$. It may happen that $d_{\lambda}(s, 0,0)$ has a different sign for different values of $s \in[0,2 \pi)$ as in the last example of $\S 3$, i.e., the family may be expanding through certain points on $L_{0}$ and contracting through other points on $L_{0}$. However, it can be shown that if $L_{0}$ is a multiple limit cycle, then if $d_{\lambda}\left(s_{0}, 0,0\right)=0$ for some $s_{0} \in \mathbf{R}, d_{\lambda}(s, 0,0)=0$ for all $s \in \mathbf{R}$. The proof of this statement is given in [23]. It then follows from the continuity of $d_{\lambda}(s, 0,0)$ that if $d_{\lambda}\left(s_{0}, 0,0\right)>0$ for some $s_{0} \in \mathbf{R}$, then $d_{\lambda}(s, 0,0)>0$ for all $s \in \mathbf{R}$. In view of these facts, we can define two types of multiple limit cycles: a multiple limit cycle $L_{0}$ of $\left(1_{0}\right)$ is called singular if $d_{\lambda}(s, 0,0)=0$ for all $s \in \mathbf{R}$ and is called nonsingular if $d_{\lambda}(s, 0,0) \neq 0$ for all $s \in \mathbf{R}$.

One immediate consequence of the above formula for $d_{\lambda}(0,0)$ is that $d_{\lambda}(0,0) \neq 0$ in any family of rotated vector fields $\left(1_{\lambda}\right)$. Thus, similar to Lemma 1.2, it follows from the implicit function theorem that if $\left(1_{\lambda}\right)$ describes a family of rotated vector fields, there exists a unique analytic function $\lambda(n)$ such that $d(n, \lambda(n))=0$ and

$$
\lambda^{\prime}(n)=-d_{n}(n, \lambda(n)) / d_{\lambda}(n, \lambda(n))
$$

for all sufficiently small $n$. It follows that in any one-parameter family of limit cycles generated by a family of rotated vector fields $\left(1_{\lambda}\right)$, only jug-handle bifurcations are possible and the family of limit cycles does not "come back on itself" as in the last example in $\S 3$. This implies that there are no cyclic families generated by a family of rotated vector fields.

Finally, it should be remarked that it is not necessary to require that the determinant $D$ defined above remain positive (or negative) in order to obtain the monotonic growth of limit cycles as in Duff [1]. It is sufficient that the integrals $I_{s}$, defined in the above remark, be positive (or negative) for all $s \in[0,2 \pi$ ) in order to establish that the one-parameter family of limit cycles generated by $\left(1_{\lambda}\right)$ expands or contracts monotonically with $\lambda$ through $L_{0}$. In fact, since it follows from equation (3) that $\operatorname{sgn}\left(d_{n}(0,0)\right)=\sigma_{0}$, where $\sigma_{0}= \pm 1$ according to whether $L_{0}$ is unstable or stable, respectively, it follows from the above equations for $\lambda^{\prime}(0)$ and $d_{\lambda}(0,0)$ (and the corresponding equations for $d_{\lambda}(s, 0,0)$ and $\left.\lambda^{\prime}(s, 0)\right)$ that if $I_{s}>0$ for $s \in[0,2 \pi)$ then $\operatorname{sgn}\left(\lambda^{\prime}(s, 0)\right)=\sigma_{0} \omega_{0}$ for all $s \in[0,2 \pi)$. Hence, if $I_{s}>0$ for all $s \in[0,2 \pi)$ then the one-parameter family of limit cycles expands through the simple limit cycle $L_{0}$ as $\lambda$ increases $(\uparrow)$ or 
as $\lambda$ decreases ( $\downarrow$ ) according to the following table:

\begin{tabular}{|l|r|r|r|r|}
\hline$\omega_{0}$ & + & + & - & - \\
\hline$\sigma_{0}$ & + & - & + & - \\
\hline$\lambda$ & $\uparrow$ & $\downarrow$ & $\downarrow$ & $\uparrow$ \\
\hline
\end{tabular}

A similar result, obtained by reversing the symbols in any one row in the above table, holds if $I_{s}<0$ for all $s \in[0,2 \pi)$. These resul, generalize the results summarized in Table 1 on p. 21 of Duff [1] and the results in the table on p. 70 in [7]. Similar results also hold for multiple limit cycles, where, in view of the above remark, it is necessary only to require that $d_{\lambda}(0,0)$ or equivalently $I_{0}$ be nonzero. These results are summarized in the following theorem, where in part (2) $\sigma_{0}$ is determined by the exterior stability of the semistable limit cycle $L_{0}$.

Theorem. If $L_{0}$ is a multiple limit cycle of $\left(1_{0}\right)$ of multiplicity $k>1$, if $I_{0}>0$, and

(1) if $k$ is odd, then $L_{0}$ belongs to a one-parameter family of limit cycles of $\left(1_{\lambda}\right)$ which expands or contracts monotonically with $\lambda$ according to the above table,

(2) if $k$ is even, then $L_{0}$ bifurcates into a stable and an unstable limit cycle as $\lambda$ varies in one sense, as described in the above table, and $L_{0}$ disappears as $\lambda$ varies in the opposite sense.

This theorem generalizes the results contained in Theorem 8, p. 23 in [1]; the results contained in Theorems 71 and 72, pp. 399 and 400 in [6]; and the result contained in Theorem F, p. 70 in [7]. It is an important result because it describes the behavior of any global family of limit cycles in the neighborhood of a nonsingular, multiple limit cycle $L_{0}$ of $\left(1_{0}\right)$. In this case, the global family has only one branch and the behavior of the corresponding one-parameter family of limit cycles is exactly the same as the behavior of a one-parameter family of limit cycles generated by a family of rotated vector fields. In the general case, suppose that $L_{0}$ is a multiple limit cycle of $\left(1_{0}\right)$ which belongs to a one-parameter family of limit cycles $\left(\lambda, L_{\lambda}\right)$ corresponding to a branch $n_{j}(\lambda)$ of the limit cycle function $n(\lambda)$, as defined in $\S 1$. Then there exist a $\delta>0$ and unique, relatively prime, positive integers $m$ and $k$ such that $n_{j}(\lambda)$ has a Puiseux series of the form

$$
n_{j}(\lambda)=(\sigma \lambda)^{m / k} \sum_{i=0}^{\infty} a_{i}(\sigma \lambda)^{i / k}
$$

which converges for $0 \leq \sigma \lambda<\sigma \delta$ where $\sigma$ is either \pm 1 . In this case $L_{0}$ is said to be a multiple limit cycle of the one-parameter family of limit cycles $\left(\lambda, L_{\lambda}\right)$ of reduced multiplicity $k$ and we have the following result: see [23]. If $k$ is even, then $L_{0}$ bifurcates into a stable and an unstable limit cycle as $\sigma \lambda$ increases; 
if $k$ is odd and $m$ is odd, then the limit cycles $L_{\lambda}$ of the family expand or contract along $L_{0}$ as $\lambda$ increases through $\lambda=0$ according to whether $\sigma a_{0}$ is positive or negative, respectively; and if $k$ is odd and $m$ is even, then the limit cycles $L_{\lambda}$ of the family expand or contract along $L_{0}$ as $\lambda$ increases in $[0, \delta)$ according to whether $a_{0}$ is positive or negative, respectively, and they expand or contract along $L_{0}$ as $\lambda$ increases in $(-\delta, 0]$ according to whether $a_{0}$ is negative or positive, respectively.

Remark 2. If $L_{0}$ is a cycle which belongs to a continuous band of cycles, then $d_{n}^{(k)}(0,0)=0$ for $k=0,1,2, \ldots$. Since $d(n, \lambda)$ is analytic in a neighborhood of $(0,0)$, it follows that

$$
d(n, \lambda)=\sum_{j=0}^{\infty} a_{j}(\lambda) n^{j}
$$

where the $a_{j}(\lambda)$ are analytic in a neighborhood of $\lambda=0$ and satisfy $a_{j}(0)=$ 0 for $j=0,1,2, \ldots$ If for $j=0,1,2, \ldots$ we have $a_{j}(\lambda) \equiv 0$ in a neighborhood of $\lambda=0$, then $L_{0}$ does not belong to any one-parameter family of limit cycles of $\left(1_{\lambda}\right)$. On the other hand, if there is a subset $J$ of the positive integers such that for $j \in J, a_{j}(\lambda) \not \equiv 0$ in a neighborhood of $\lambda=0$, then

$$
a_{j}(\lambda)=\lambda^{k_{j}} \sum_{i=0}^{\infty} b_{i j} \lambda^{i}
$$

in a neighborhood of $\lambda=0$ where for all $j \in J, b_{0 j} \neq 0$ and $k_{j} \geq 1$. Now let $k^{*}=\min _{j} k_{j}$ and let $j^{*}$ be the smallest positive integer $j \in J$ such that $k_{j}=k^{*}$. Then $k^{*} \geq 1$ and

$$
d(n, \lambda)=\lambda^{k^{*}} \sum_{j \in J} \lambda^{k_{j}-k^{*}} \sum_{i=0}^{\infty} b_{i j} \lambda^{i} n^{j}
$$

in a neighborhood of the origin where $k_{j}-k^{*} \geq 0$ and $k_{j^{*}}-k^{*}=0$. It follows that at $(n, \lambda)=(0,0)$ we have

$$
\frac{\partial^{j^{*}}}{\partial n^{j^{*}}}\left[\frac{d(n, \lambda)}{\lambda^{k^{*}}}\right]=\left(j^{*}\right) ! b_{0 j^{*}} \neq 0
$$

Thus, just as in $\S 1$ of this paper, the Weierstrass preparation theorem, applied to the function $d(n, \lambda) / \lambda^{k^{*}}$, implies that the relation $d(n, \lambda)=0$ has at most $j^{*}$ real branches which define local one-parameter families of limit cycles passing through $L_{0}$ at $\lambda=\lambda_{0}$.

Acknowledgment. This work was supported by a Northern Arizona University Organized Research Grant.

\section{REFERENCES}

1. G. F. D. Duff, Limit cycles and rotated vector fields, Ann. of Math. 67 (1953), 15-31.

2. A. Wintner, Beweis des E. Stromgrenschen dynamischen Abschlusprinzips der periodischen Bahngruppen im restringierten Dreikorperproblem, Math. Z. 34 (1931), 321-349. 
3. L. M. Perko, On the accumulation of limit cycles, Proc. Amer. Math. Soc. 99 (1987), 515526.

4. J. P. Francoise and C. C. Pugh, Keeping track of limit cycles, J. Differential Equations 65 (1986), 139-157.

5. C. Sparrow, The Lorentz equations: bifurcations, chaos, and strange attractors, Appl. Math. Sci., vol. 41, Springer-Verlag, New York, 1982.

6. A. A. Andronov, E. A. Leontovich, I. I. Gordon, and A. G. Maier, Theory of bifurcations of dynamical systems on a plane, Israel Program for Scientific Translations, Jerusalem, 1971.

7. L. M. Perko, Rotated vector fields and the global behavior of limit cycles for a class of quadratic systems in the plane, J. Differential Equations 18 (1975), 63-86.

8. J. Mallet-Paret and J. A. Yorke, Snakes: oriented families of periodic orbits, their sources, sinks and continuation, J. Differential Equations 43 (1982), 419-450.

9. K. T. Alligood and J. A. Yorke, Families of periodic orbits: virtual periods and global continuity, J. Differential Equations 55 (1984), 59-71.

10. S. N. Chow, J. Mallet-Paret, and J. A. Yorke, A bifurcation invariant: degenerate orbits treated as clusters of simple orbits, Lecture Notes in Math., vol. 1007, Springer-Verlag, Berlin, 1983, pp. 109-131.

11. K. T. Alligood, J. Mallet-Paret, and J. A. Yorke, An index for the global continuation of relatively isolated sets of periodic orbits, Lecture Notes in Math., vol. 1007, Springer-Verlag, Berlin, 1983, pp. 1-21.

12. H. Poincaré, Mémoire sur les courbes définies par une equation différentiell, J. Mathematiques 7 (1881), 375-422; Oeuvre, Gauthier-Villar, Paris, 1880-1890.

13. J. Guckenheimer and P. Holmes, Nonlinear oscillations, dynamical systems and bifurcations of vector fields, Appl. Math. Sci., vol. 42, Springer-Verlag, New York, 1983.

14. J. Dieudonné, Foundations of modern analysis, Academic Press, New York, 1960.

15. E. A. Coddington and N. Levinson, Theory of ordinary differential equations, McGraw-Hill, New York. 1955.

16. M. W. Hirsch and S. Smale, Differential equations, dynamical systems and linear algebra, Academic Press, New York, 1974.

17. L. M. Graves, The theory of functions of real variables, McGraw-Hill, New York, 1956.

18. A. A. Andronov et al., Qualitative theory of second order dynamical systems, Wiley, New York, 1973.

19. I. Bendixson, Sur les courbes définies par des équations différentielles, Acta Math. 24 (1901), $1-88$.

20. L. M. Perko and S. S. Lung, Existence, uniqueness, and nonexistence of limit cycles for a class of quadratic systems in the plane, J. Differential Equations 53 (1984), 146-171.

21. B. Coll, A. Gasull, and J. L. Libre, Some theorems on the existence, uniqueness, and nonexistence of limit cycles for quadratic systems, J. Differential Equations 67 (1987), 372-399.

22. L. M. Perko, Bifurcation of limit cycles. Proc. Conference on Bifurcations and Periodic Orbits of Planar Vector Fields, Luminy, France, September 1989; Lecture Notes in Math., Springer-Verlag, (to appear).

23. L. M. Perko, Bifurcation of limit cycles: geometric theory, Proc. Amer. Math. Soc. (submitted).

Department of Mathematics, Northern arizona University, Flagstaff, Arizona $86011-5717$ 